This is a post-print of the following article: Andric, Predrag; Yin, Binglun; Curtin, W.A. Journal of the Mechanics and Physics of Solids 2019, , 262-279.. The formal publication is available at http://dx.doi.org/10.1016/j.jmps.2018.09.007 (C) 2019. This manuscript version is made available under the CC-BY-NC-ND 4.0 license http://creativecommons.org/licenses/by-nc-nd/4.0/

\title{
Stress-dependence of generalized stacking fault energies
}

\author{
Predrag Andric, Binglun Yin, W.A. Curtin \\ Laboratory for Multiscale Mechanics Modeling \\ Ecole Polytechnique Fédérale de Lausanne \\ CH-1015 Lausanne, Switzerland
}

\section{Abstract}

The energy associated with shearing of planes of atoms in a crystal is the generalized stacking fault energy (GSFE). It is a crucial material property for describing nanoscale plasticity phenomena in crystalline materials, such as dislocation dissociation, nucleation, and twinning. The dependence of the GSFE on applied stress normal to the stacking fault has been suggested to influence such phenomena. Here, the stacking fault stress dependence is analyzed through (i) the generalized stacking fault potential energy (GSFE) and (ii) the generalized stacking fault enthalpy (GSFH). At an imposed shear displacement, there is also an associated inelastic inter-planar normal displacement around the fault. Extensive molecular statics simulations with interatomic potentials and/or first principle calculations in $\mathrm{Ni}, \mathrm{Cu}, \mathrm{Al}$ and $\mathrm{Mg}$ reveal that GSFE and inelastic normal displacement both increase with tensile stress. An increasing GSFE contradicts long-standing wisdom and previous studies. Positive inelastic normal displacement coupled to the applied normal stress decreases the GSFH, but is not useful for general mechanics problems. The existence of the inelastic displacement can lead to incorrect measurements of the GSFE and GSFH in finite systems loaded by an applied strain. Application of the GSFE and the inelastic normal displacement to both fcc dissociation distance versus applied normal stress and crack tip dislocation emission under mixed Mode II/I loading show very good agreement with direct simulations. In general, “opening softening” effects are not universal, and so the analysis of any particular nanomechanics problem requires precise implementation of the combination of GSFE and inelastic normal displacement rather than the GSFH.

30 Keywords: Generalized Stacking Fault Energy, Molecular Statics, First Principle Calculations, Opening Softening, Dislocations. 
This is a post-print of the following article: Andric, Predrag; Yin, Binglun; Curtin, W.A. Journal of the Mechanics and Physics of Solids $2019,, 262-279$.. The formal publication is available at http://dx.doi.org/10.1016/j.jmps.2018.09.007 () 2019. This manuscript version is made available under the CC-BY-NC-ND 4.0 license http://creativecommons.org/licenses/by-nc-nd/4.0/

\section{Introduction}

The Generalized Stacking Fault Energy (GSFE) $\Psi_{g s f}$ is one of the most important properties

35 for understanding dislocation phenomena in crystalline materials [1]. The GSFE governs the dissociation distance of fcc partial dislocations, the distribution of Burgers vector across the core of a dislocation, and is used in the classic Peierls-Nabarro model to compute these physical features [2]. The GSFE is also recognized as a crucial material property for describing nanoscale plasticity, especially dislocation nucleation processes such as dislocation emission (i) from a crack tip [3, 4],

40 (ii) from a grain boundary [5, 6], (iii) during nanoindentation [7], and (iv) to create crack-tip twinning $[8,9]$.

For a given slip plane and slip direction in a crystal, the GSFE is the energy associated with an imposed shear displacement $\Delta_{S}$ between two non-sheared crystalline blocks. The standard measurement is performed allowing displacements of all atoms normal to the slip plane, and thus full relaxation of the normal stress (or traction) $T_{n}$ throughout the cell, leading to $\Psi_{g s f}\left(\Delta_{s}, T_{n}=0\right)$. The normal relaxation gives rise to changes in the inter-planar spacings around the fault; we denote the total inelastic normal displacement as $\Delta_{n}$. The changes in planar spacings and $\Delta_{n}$ are often neglected. In some early mechanics works, computations held the two blocks rigidly at the unstrained lattice constant of the crystal and then minimized the energy by relaxing the normal displacement across only the two planes of atoms on either side of the fault plane [10]. In this case, $\Delta_{n}$ is slightly different than in the more-general case and is localized to the fault plane, but again this is often neglected.

In fcc metals, the focus here, favorable sliding occurs along the $\langle 112\rangle$ crystallographic direction. The GSFE has a local minimum at $\Delta_{s}=b_{p}$ where $b_{p}=a_{0} / \sqrt{6}$ is the magnitude of the partial dislocation Burgers vector for fcc lattice parameter $a_{0}$. The GSFE at this point is the stable stacking fault energy $\gamma_{s s f}$. Prior to reaching the stable stacking fault, the GSFE has maximum corresponding to the unstable stacking fault energy with energy $\gamma_{u s f}$. The quantities $\gamma_{s s f}$ and $\gamma_{u s f}$ are primary fault energies used to understand various dislocation phenomena in metals. Typical GSFE curves for $\mathrm{Al}$ [11], with full normal relaxation and relaxation only across the slip plane, both at $T_{n}=0$, are shown in Figure 1. The difference between the two methods is indeed small in this case, largely justifying the prior neglect of the differences between the two methods.

The high stresses often associated with nanoscale phenomena suggest that the normal stress dependence of the GSFE, and in particular of $\gamma_{s s f}$ and/or $\gamma_{u s f}$, are important. Rice and collaborators postulated that $\gamma_{u s f}$ decreases under a high tensile stress $T_{n}$, facilitating crack tip dislocation emission $[3,10]$. Such "opening softening" was estimated to decrease the critical energy release rate for crack65 tip dislocation emission $G_{I e}$ by up to $30 \%$ in fcc metals [10]. Subsequently, the notion of "opening softening" was invoked in different contexts but without a quantitative analysis of its effects in each 
This is a post-print of the following article: Andric, Predrag; Yin, Binglun; Curtin, W.A. Journal of the Mechanics and Physics of Solids 2019, , 262-279.. The formal publication is available at http://dx.doi.org/10.1016/j.jmps.2018.09.007 (C) 2019. This manuscript version is made available under the CC-BY-NC-ND 4.0 license http://creativecommons.org/licenses/by-nc-nd/4.0/

particular problem $[12,13]$. The need for quantification motivated several computational studies of the stress effects on the GSFE using molecular statics simulations [14, 15] and first principle calculations $[16,17]$. All of these studies reported "opening softening". The prior computational studies were performed under displacement boundary conditions, which we show below can be problematic. In addition, the Rice theory for dislocation emission is typically somewhat lower than detailed simulation studies [4] and so "opening softening" would lead to further deviations between theory and simulation. The present authors also recently introduced a new theory/analysis for cracktip dislocation emission and twinning that agrees very well with simulations of the critical stress intensity factor $K_{I e}$ without including any "opening softening" effect [4, 9]. These factors motivate us to revisit the measurement/computation of the stress dependence of the GSFE more thoroughly than in earlier works.

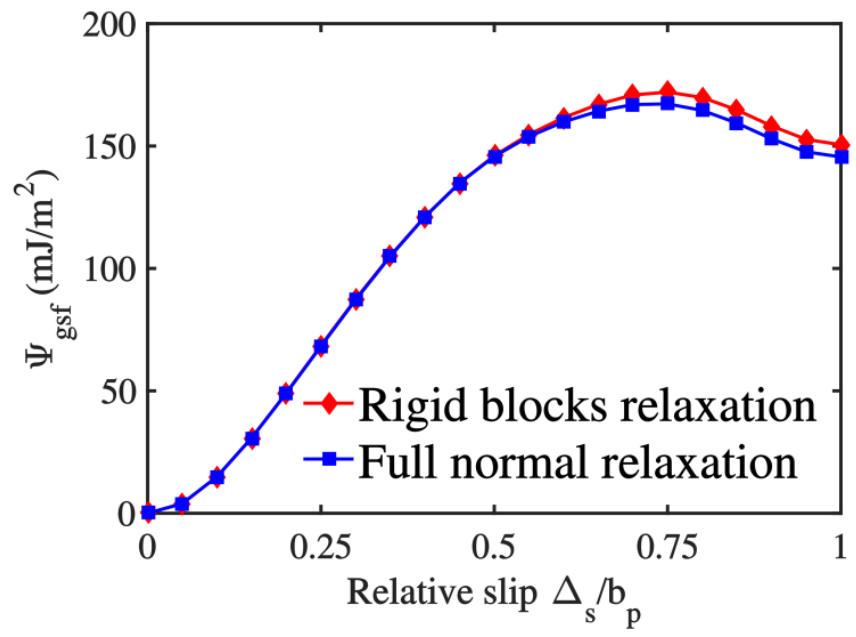

Figure 1: Generalized stacking fault energy with full normal relaxation (blue squares) and relaxation only across the slip plane (red diamonds) computed in Aluminum [11] at $\mathrm{T}=0 \mathrm{~K}$.

GSF calculations under an applied stress can be envisioned in principle through two thermodynamic interface quantities, (i) a generalized stacking fault (potential) energy GSFE, and (ii) a generalized stacking fault enthalpy (GSFH, consistent with the standard thermodynamic notation for the enthalpy as $\mathrm{H}=\mathrm{E}+\mathrm{PV})$. The $\operatorname{GSFE} \Psi_{g s f}\left(\Delta_{s}, \Delta_{n}\right)$ is a local surface constitutive law associated with shearing $\Delta_{s}$ and normal displacement $\Delta_{n}$. However, the total inelastic normal displacement observed under applied normal stress $\Delta_{n}\left(\Delta_{s}, T_{n}\right)$ extends over several atomic planes around the stacking fault and so is non-local. A $\Delta_{n}$ cannot easily be imposed since there are actually individual internal variables $\Delta_{n}^{(i)}$ for successive planar spacings $\{i=0, \pm 1, \pm 2, \ldots\}$. By changing variables from normal displacement to normal traction, we can define $\Psi_{g s f}\left(\Delta_{s}, \Delta_{n}\left(T_{n}\right)\right) \equiv \Psi_{g s f}\left(\Delta_{s}, T_{n}\right)$ also as the GSFE (potential energy). Under normal traction, not all interatomic planar spacings $\left\{\Delta_{n}^{(i)}\right\}$ are accessible; only those corresponding to the imposed traction $T_{n}$ can be measured. As noted above, 
This is a post-print of the following article: Andric, Predrag; Yin, Binglun; Curtin, W.A. Journal of the Mechanics and Physics of Solids $2019,, 262-279$.. The formal publication is available at http://dx.doi.org/10.1016/j.jmps.2018.09.007 @ 2019. This manuscript version is made available under the CC-BY-NC-ND 4.0 license http://creativecommons.org/licenses/by-nc-nd/4.0/

90 the standard GSFE is precisely $\Psi_{g s f}\left(\Delta_{s}, \Delta_{n}\left(T_{n}=0\right)\right) \equiv \Psi_{g s f}\left(\Delta_{s}, T_{n}=0\right)$ and the value of $\Delta_{n}\left(T_{n}=0\right)$ is not usually reported. Here, we apply a normal traction $T_{n}$ and directly measure $\Psi_{g s f}\left(\Delta_{s}, T_{n}\right)$ and $\Delta_{n}\left(\Delta_{s}, T_{n}\right)$. The GSFE $\Psi_{g s f}\left(\Delta_{s}, T_{n}\right)$ generally increases, or remains nearly constant, over a wide range of tractions approaching normal decohesion levels; there is no "opening softening".

Under an applied traction normal to the fault plane, the relevant thermodynamic quantity for the entire system is the enthalpy. Subtracting the enthalpy of the reference perfect crystal yields the GSFH, $\Psi_{g s f}^{\text {enth }}\left(\Delta_{s}, T_{n}\right)=\Psi_{g s f}\left(\Delta_{s}, T_{n}\right)-T_{n} \Delta_{n}\left(\Delta_{s}, T_{n}\right)$. When the normal opening is positive, this quantity can decrease under increasing normal stress. Previously reported "opening softening" results are essentially calculations of $\Psi_{g s f}^{\text {enth }}$ [14-17] (aside from detrimental finite size effects). However, the term "enthalpy" was never used, and the quantity was often reported as the GSFE (potential energy) under stress. The distinction between GSFE and GSFH is important. While $\Psi_{g s f}^{e n t h}$ is the proper thermodynamic quantity for the entire system under a traction normal to an infinite fault plane, this quantity cannot easily be used in general mechanics problems and, if used improperly can lead to spurious conclusions.

The importance of defining and distinguishing the various thermodynamic quantitates in mechanics problems is thus highlighted here in two applications: (i) fcc partial dissociation under applied normal stress, and (ii) crack-tip dislocation emission under mixed Mode II/I loading. We show that a precise use and/or understanding of $\Psi_{g s f}\left(\Delta_{s}, T_{n}\right)$ and $\Delta_{n}\left(\Delta_{s}, T_{n}\right)$ enables quantitative understanding of explicit molecular statics simulations of these two problems. In particular, for cracktip dislocation emission, we demonstrate that there is no "opening softening" as previously reported and the simulations are entirely consistent with the underlying $\Psi_{g s f}\left(\Delta_{s}, T_{n}\right)$ and $\Delta_{n}\left(\Delta_{s}, T_{n}\right)$.

The remainder of this paper is organized as follows. In Section 2, we introduce the methods for computing the GSFE and inelastic normal displacement under an applied normal tensile stress. Section 3 presents our results for the GSFE and inelastic normal displacement, as computed using interatomic potentials and first-principles methods, and the subsequent determinations of $115 \Psi_{g s f}\left(\Delta_{s}, \Delta_{n}\right)$ and $\Psi_{g s f}^{e n t h}\left(\Delta_{s}, T_{n}\right)$. In Section 4, we show the errors that arise in the computation of the GSFE and GSFH when computed using displacement boundary condition; these rationalize previous literature results. In Section 5.1, we present the analysis of mechanics problems using the GSFE and inelastic normal displacements with the framework of the Eshelby method for defects in infinite elastic bodies. Section 5.2 presents the analysis and results of partial dislocation separation distance versus normal stress fcc metals. Section 5.3 presents results and analysis of crack-tip dislocation emission in mixed mode II/I loading. Finally, in Section 6, we discuss the general implications of our results. 
This is a post-print of the following article: Andric, Predrag; Yin, Binglun; Curtin, W.A. Journal of the Mechanics and Physics of Solids 2019,, 262-279.. The formal publication is available at http://dx.doi.org/10.1016/j.jmps.2018.09.007 @ 2019. This manuscript version is made available under the CC-BY-NC-ND 4.0 license http://creativecommons.org/licenses/by-nc-nd/4.0/

\section{GSFE under normal stress: simulation methods}

We compute the GSFE under applied normal stress using interatomic potentials (and the LAMMPS code [18]) and first principles density-functional theory (DFT) calculations (VASP code [19-20]) in molecular statics. The GSFE for relative sliding of two crystalline blocks of material is computed in the standard manner (see Figure 2). At relative shear displacement $\Delta_{s}$, the GSFE is computed as

$$
\Psi_{g s f}\left(\Delta_{s}, T_{n}\right)=\left(\mathrm{E}\left(\Delta_{s}, T_{n}\right)-\mathrm{E}\left(\Delta_{s}=0, T_{n}\right)\right) / A
$$

130 where $\mathrm{E}\left(\Delta_{s}=0, T_{n}\right)$ is the potential energy of a perfect crystal under stress $T_{n}, \mathrm{E}\left(\Delta_{s}, T_{n}\right)$ is the potential energy of the simulation cell at relative slip displacement $0 \leq \Delta_{s} \leq b_{p}$, and A is the area of the simulated fault. Note that in the above, the work done by the applied stress is not included, so that Eq. 1 is the desired potential energy.
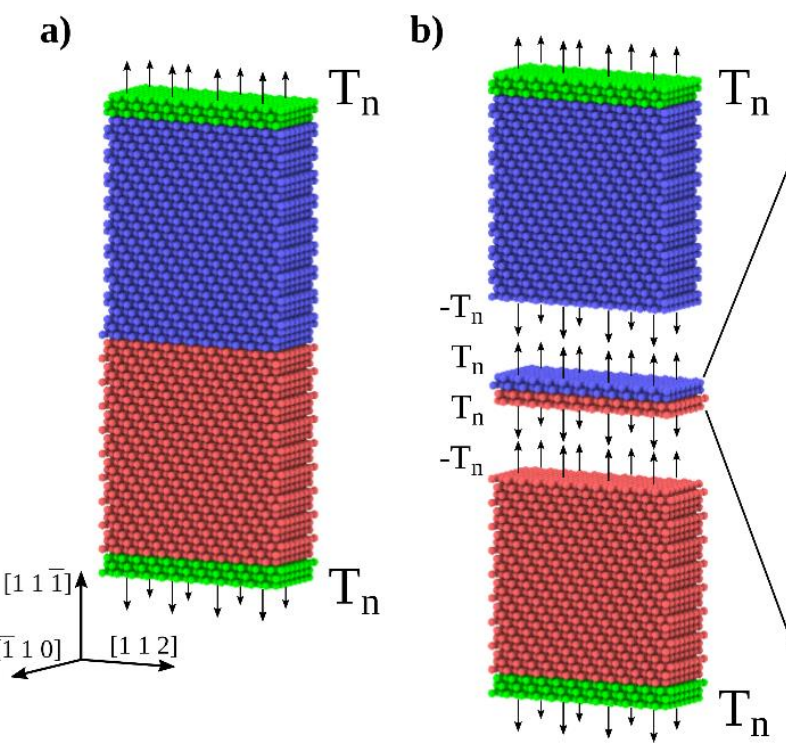

c)

Figure 2: a) Typical simulation cell for computing the GSFE under applied normal stress, with the lower and upper domains indicated by red and blue, and atoms on which forces are applied indicated in green. b) Stacking fault region for describing the local constitutive behavior for rigid block sliding under applied tractions. c) Inter-planar spacing around the stacking fault for a given shear displacement $\Delta_{s}$ and applied tractions $T_{n}$. Atoms are visualized using OVITO [30].

In the interatomic potential computations, we first define a rectangular simulation cell oriented with $X=[112], Y=[11 \overline{1}]$ and $Z=[\overline{1} 10]$, and dimensions $6 \sqrt{6} a_{0} \times 20 \sqrt{3} a_{0} \times 2 \sqrt{2} a_{0}$. We set periodic boundary conditions along the $\mathrm{X}$ and $\mathrm{Z}$ direction in the plane of the fault, and traction boundary conditions in the $\mathrm{Y}$ direction normal to the fault. The simulation cell length in the $\mathrm{Y}$ direction is sufficient to prevent any interaction between the upper and lower surfaces and the stacking fault. The desired stress $T_{n}$ along the $\mathrm{Y}=[11 \overline{1}]$ direction is created by applying forces to atoms within $r_{c}\left(r_{c}=\right.$ cut-off distance of the interatomic potential) of the top and bottom Y boundaries (see Figure 2a). At a given applied normal stress $\sigma_{y y}=T_{n}$ we compute initial atomic positions and the initial 
This is a post-print of the following article: Andric, Predrag; Yin, Binglun; Curtin, W.A. Journal of the Mechanics and Physics of Solids 2019,, 262-279.. The formal publication is available at http://dx.doi.org/10.1016/j.jmps.2018.09.007 @ 2019. This manuscript version is made available under the CC-BY-NC-ND 4.0 license http://creativecommons.org/licenses/by-nc-nd/4.0/

145 load $T_{n}$, the cell sizes along $\mathrm{X}$ and $\mathrm{Z}$ are held fixed; thus lateral loads $\sigma_{x x}$ and $\sigma_{z z}$ develop as $T_{n}$ is increased. However, in finite-length simulation cells, allowing relaxation of the total lateral stresses includes a compensation for lateral stresses due to the fault itself; this leads to erroneous stresses away from the fault, and changes in stored elastic energy that are then attributed to the fault only. Thus, it is best to use fixed cell sizes along $\mathrm{X}$ and $\mathrm{Z}$ with periodic boundary conditions; the GSFE under uniaxial stress is shown in Appendix A for comparison and differences are negligible. After the initial minimization, the upper half of the crystal in the [112] slip direction is displaced relative to the (fixed) lower half by the displacement $\Delta_{s}$. The applied normal stress is held fixed for all shear displacement values. As with standard GSF computations, atomic relaxation is allowed only in the Y direction normal to the slip plane and the minimum energy computed. We perform calculations in fcc Nickel, Copper and Aluminum using the Mishin et al. EAM interatomic interactions [11, 22].

First principle DFT calculations of the GSFE in Cooper, Aluminum and Magnesium are performed as follows. The exchange-correlation functional is treated within the generalized gradient approximation (GGA) with the Perdew-Burke-Ernzerhof (PBE) parametrization [23]. Core electrons are replaced by the projector augmented wave (PAW) pseudopotentials [24]. The cutoff energy of the plane wave basis set is $800 \mathrm{eV}$ for $\mathrm{Cu}, 500 \mathrm{eV}$ for $\mathrm{Al}$, and $400 \mathrm{eV}$ for $\mathrm{Mg}$, respectively. Simulation of the GSFE is accomplished using the tilted-cell method [25]. We start with a simulation cell in the fully-periodic bulk crystal structure with the X-Z plane parallel to the desired stacking fault plane. The (non-orthogonal) cell has lattice vectors $\left(\boldsymbol{a}_{\boldsymbol{x}}, \boldsymbol{a}_{\boldsymbol{y}}, \boldsymbol{a}_{z}\right)$. A new periodic simulation cell with lattice vectors $\left(\boldsymbol{a}_{\boldsymbol{x}}, \boldsymbol{a}_{\boldsymbol{y}}+\Delta_{s} \boldsymbol{t}, \boldsymbol{a}_{z}\right)$ is then defined where $\boldsymbol{t}$ is a unit vector lying in the X-Z slip plane and in the direction of the slip. The change in periodicity thus introduces an initial atomistic fault across the $\mathrm{X}-\mathrm{Z}$ periodic boundary of the cell. All atoms are then permitted to relaxed in the $\mathrm{Y}$ direction normal to the fault and the length of the cell normal to the fault plane is also allowed to relax such that the normal stress is the desired applied stress, $\sigma_{y y}=T_{n}$. This procedure is appropriate for computing the GSFE when atoms undergo no in-plane relaxations, which is true by symmetry for the fcc stable stacking fault position and is generally valid for fcc and hep basal fault planes (see [26] for notable exceptions for other hcp slip planes). We use 12 atomic layers normal to the fault, which is just about sufficient to avoid SF-SF interactions of the periodic images for the close-packed planes in $\mathrm{Cu}, \mathrm{Al}$, and $\mathrm{Mg}$. Our results for the stable stacking fault energies are then close to those reported in other work [26] using different methods and different cell sizes. Other details of the DFT parameters and geometry setup can be found in Ref. [26].

We now introduce few technical points that are crucial for achieving accurate DFT results. First, the force convergence criterion must be selected carefully. In some cases, it must be smaller than the value of $10^{-3} \mathrm{eV} / \AA ̊ \AA$ typically used in DFT studies. The smaller value is necessary due to 
This is a post-print of the following article: Andric, Predrag; Yin, Binglun; Curtin, W.A. Journal of the Mechanics and Physics of Solids $2019,, 262-279$.. The formal publication is available at http://dx.doi.org/10.1016/j.jmps.2018.09.007 @ 2019. This manuscript version is made available under the CC-BY-NC-ND 4.0 license http://creativecommons.org/licenses/by-nc-nd/4.0/

energy contributions arising from the corresponding uncertainty in the stress state when stress boundary conditions are used. For an uncertainty in stress of $d \sigma$, the uncertainty in the energy is $d E=\frac{V}{2 C}\left(d \sigma^{2}+2 \sigma d \sigma\right)$ assuming linear elasticity, where $\mathrm{C}$ is the plane-strain elastic modulus for loading along the y direction. Taking $\mathrm{Mg}$ as an example $\left(C_{33}=63.6 \mathrm{GPa}\right.$, atomic volume $22.9 \AA^{3}$, 12 atoms in the supercell), in the stress-free state $\left(T_{n}=0\right)$ force convergence at $10^{-3} \mathrm{eV} / \AA ̊$ corresponds to $d \sigma \sim 7 \cdot 10^{-3} \mathrm{GPa}$ and $\mathrm{dE} \sim 7 \cdot 10^{-7} \mathrm{eV}$, which is negligible. However, at

185 a stress $T_{n}=5 \mathrm{GPa}$, the error is $\mathrm{dE} \sim 0.9 \mathrm{meV}$. Since the GSFE energy is calculated by subtracting two supercell energies, this uncertainty in the supercell total energy can cause a large uncertainty in the computed GSFE, with uncertainty of $\sim 3 \mathrm{~mJ} / \mathrm{m}^{2}$ for $\mathrm{Mg}$. Here, we choose $10^{-4} \mathrm{eV} / \AA(\sim 0.7$. $10^{-3} \mathrm{GPa}$ ) for $\mathrm{Al}$ and $\mathrm{Mg}$, reducing the total error in energy to $\sim 0.09 \mathrm{meV}$ and thus good accuracy in the computation of the GSFE. For $\mathrm{Cu}$, the effective elastic constant $\mathrm{C}$ is approximately four times 190 larger than that of $\mathrm{Mg}$ so that a force tolerance of $10^{-3} \mathrm{eV} / \mathrm{A}$ leads to an error of $0.3 \mathrm{meV}$ at $5 \mathrm{GPa}$, and hence only an error of $1.3 \mathrm{~mJ} / \mathrm{m}^{2}$ in the GSFE. Difficulty in converging of ionic relaxations in $\mathrm{Cu}$ limits the force tolerance to $10^{-3} \mathrm{eV} / \AA$ and so it is not possible to reduce the error further. This discussion also shows that a smaller number of layers introduces less error. But too few layers lead to spurious interactions among the periodic images of the SF. We find that 12 layers of atoms is satisfactory for avoiding both problems.

\section{GSFE under normal stress: results}

Figure 3(i) shows the GSFE $\Psi_{g s f}\left(\Delta_{s}, T_{n}\right)$ as computed for $\mathrm{Ni}, \mathrm{Cu}$, and $\mathrm{Al}$ using interatomic potentials for a range of applied normal tensile stresses up to $T_{n}=15 \mathrm{GPa}$. In $\mathrm{Ni}$ and $\mathrm{Cu}$, the entire GSFE curve increases with applied normal tensile stress; the increases in $\Psi_{g s f}$ up to $10 \mathrm{GPa}$ are fairly small, however. Similar trends are generally found in Al but the GSFE does decrease for the highest stress and large shear displacements, $\Psi_{g s f}\left(\Delta_{s}>0.7 b_{p}, T_{n}=7.5 \mathrm{GPa}\right)$, where the normal stress is approaching the material cohesive stress (maximum sustainable normal stress in the material). Focusing on the most important points of the GSFE, the unstable and stable stacking fault energies $\gamma_{u s f}$ and $\gamma_{s s f}$, Figure 4 shows $\gamma_{u s f}$ and $\gamma_{s s f}$ normalized by their values at $T_{n}=0$. In Ni and $\mathrm{Cu}, \gamma_{u s f}$ is fairly insensitive to stress up to $\sim 10 \mathrm{GPa}$, while $\gamma_{u s f}$ increases more rapidly in Al. In contrast, $\gamma_{s s f}$ varies more rapidly with stress, especially in $\mathrm{Al}$ and $\mathrm{Cu}$. $\mathrm{Al}$ does show "opening softening" (normalized values below unity) at $T_{n}>6 \mathrm{GPa}$. Overall, the trend for all materials is "opening hardening". 
This is a post-print of the following article: Andric, Predrag; Yin, Binglun; Curtin, W.A. Journal of the Mechanics and Physics of Solids 2019, , 262-279.. The formal publication is available at http://dx.doi.org/10.1016/j.jmps.2018.09.007 @ 2019. This manuscript version is made available under the CC-BY-NC-ND 4.0 license http://creativecommons.org/licenses/by-nc-nd/4.0/

a) nickel

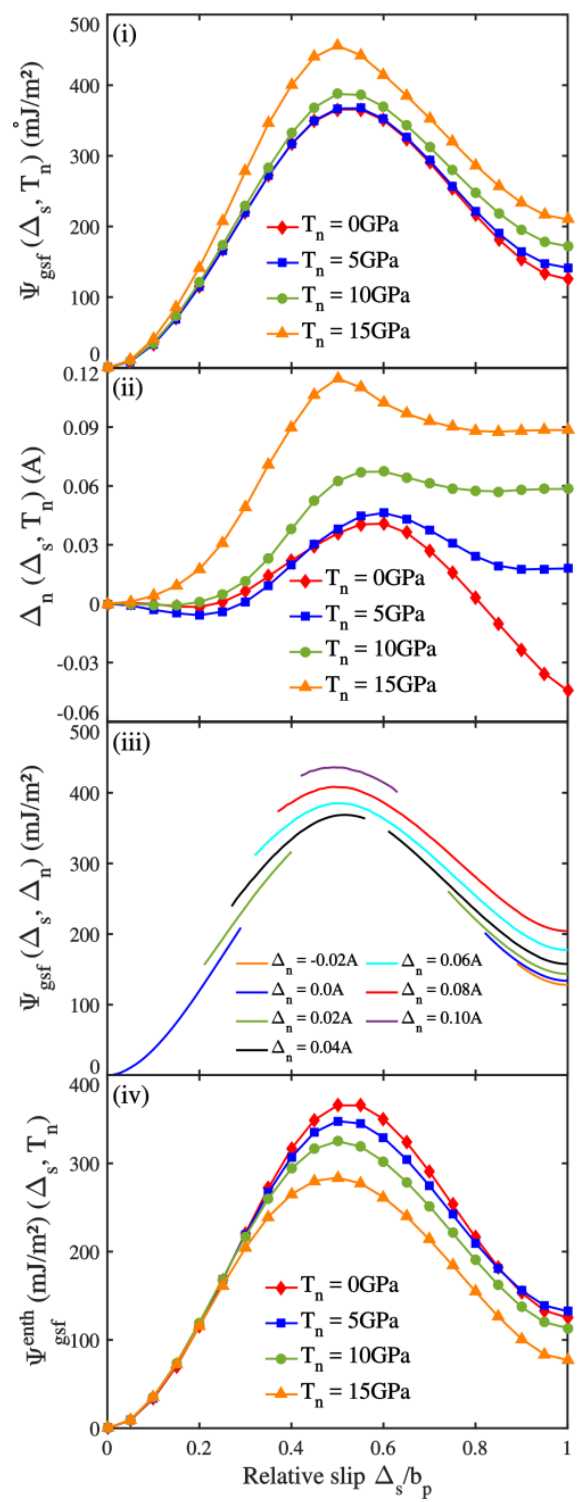

b) copper

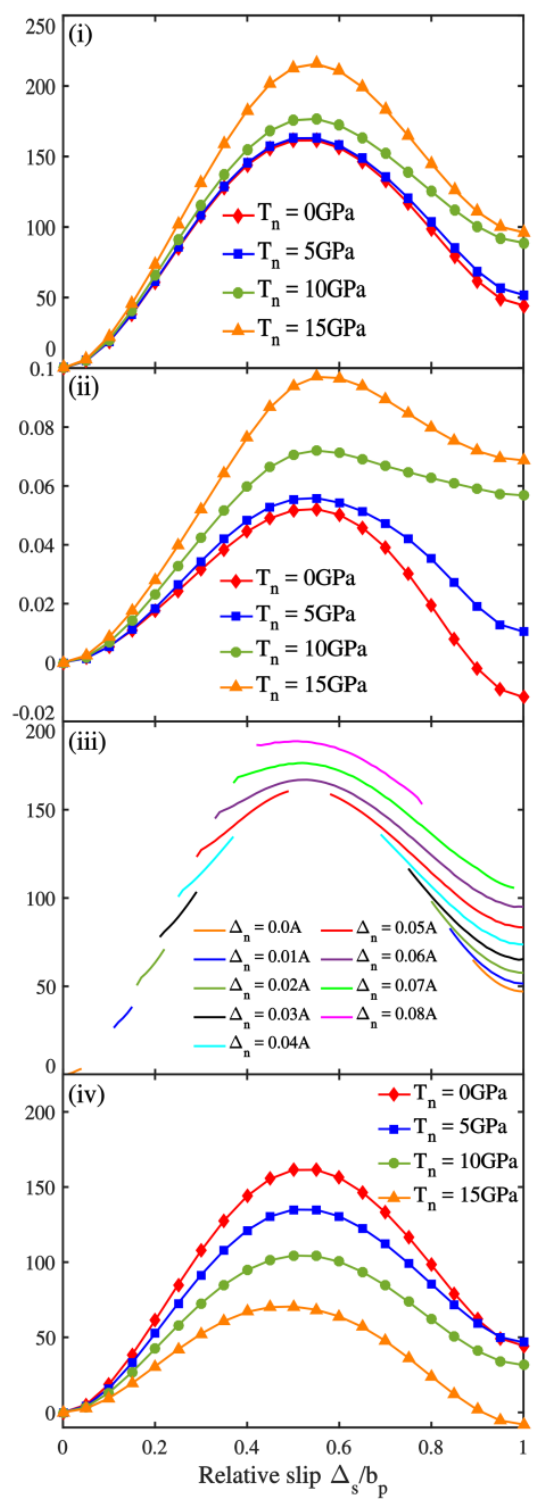

c) aluminum

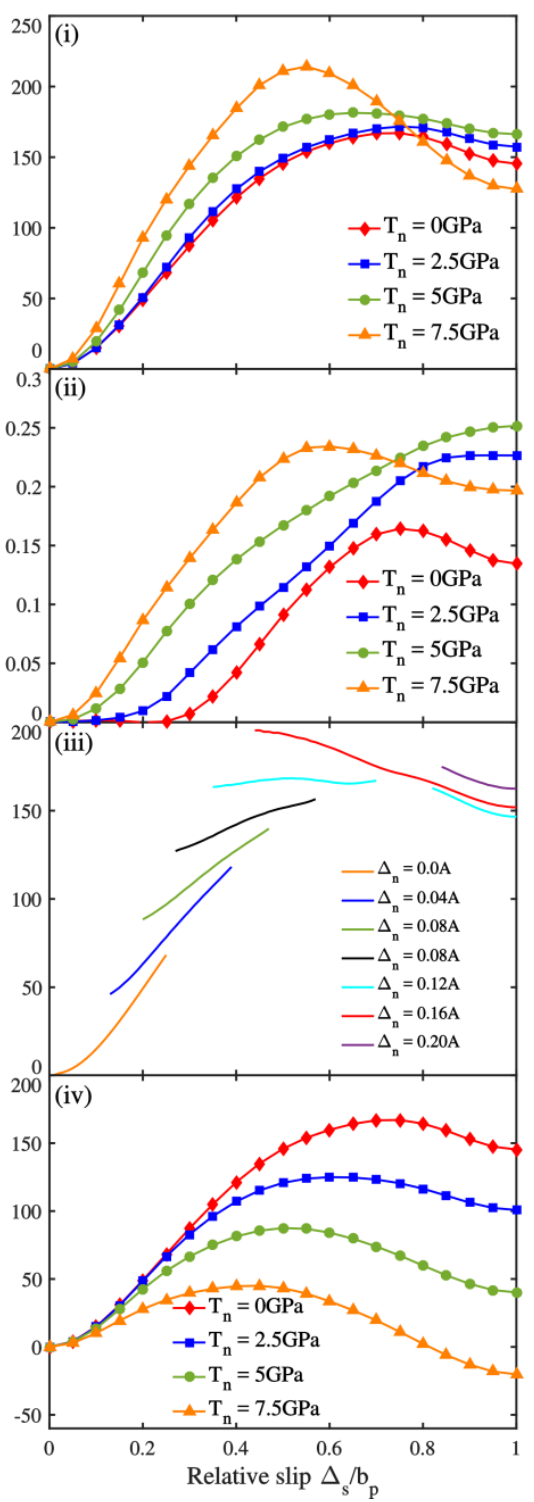

Figure 3: (i) The GSFE $\Psi_{g s f}$, and ii) total inelastic normal displacement $\Delta_{n}$ across the slip plane for different applied normal stresses $T_{n}$; (iii) The GSFE $\Psi_{g s f}$ for different applied inelastic displacements $\Delta_{n}$; (iv) GSFH versus shear displacement $\Delta_{s}$ for different applied normal stresses $T_{n}$. All values are computed with interatomic potentials at $\mathrm{T}=0 \mathrm{~K}$ in a) nickel, b) copper and c) aluminum.

Studies using EAM potentials enable us to analyze atom-by-atom energy changes around the fault. For all shear displacements, the energy contributions to the GSFE are localized to two (Ni and $\mathrm{Cu})$ or three $(\mathrm{Al})$ atomic planes on each side of the slip plane. Figure 5 shows examples at the unstable and the stable stacking fault displacements for Ni. This is fully expected since the GSFE is the energy change of the atoms due to the presence of the planar fault, and deviations from perfect crystal behavior are well-known to extend only a few layers of atoms from the defect plane even for much more drastic planar defects such as free surfaces and grain boundaries.

Figure 6(i) shows the GSFE versus applied normal stress as computed via first-principles for $\mathrm{Al}, \mathrm{Cu}$, and the basal plane in $\mathrm{Mg}$ (which is very similar to fcc). In all three materials, the qualitative trends are similar to those obtained using interatomic potentials. There is no "opening softening". For 
This is a post-print of the following article: Andric, Predrag; Yin, Binglun; Curtin, W.A. Journal of the Mechanics and Physics of Solids 2019,, 262-279.. The formal publication is available at http://dx.doi.org/10.1016/j.jmps.2018.09.007 @ 2019. This manuscript version is made available under the CC-BY-NC-ND 4.0 license http://creativecommons.org/licenses/by-nc-nd/4.0/

the stresses considered here, the DFT-computed GSFE is nearly constant in $\mathrm{Cu}$ and $\mathrm{Mg}$ and increases in Al. Quantitative differences between DFT and interatomic potentials are expected, with the DFT being the reference, but the differences are not significant relative to our main points. These results also show that the GSFE typically increases with applied stress. There is no "opening softening", in contrast to prior concepts and results in this literature. This is the first main result of this paper.
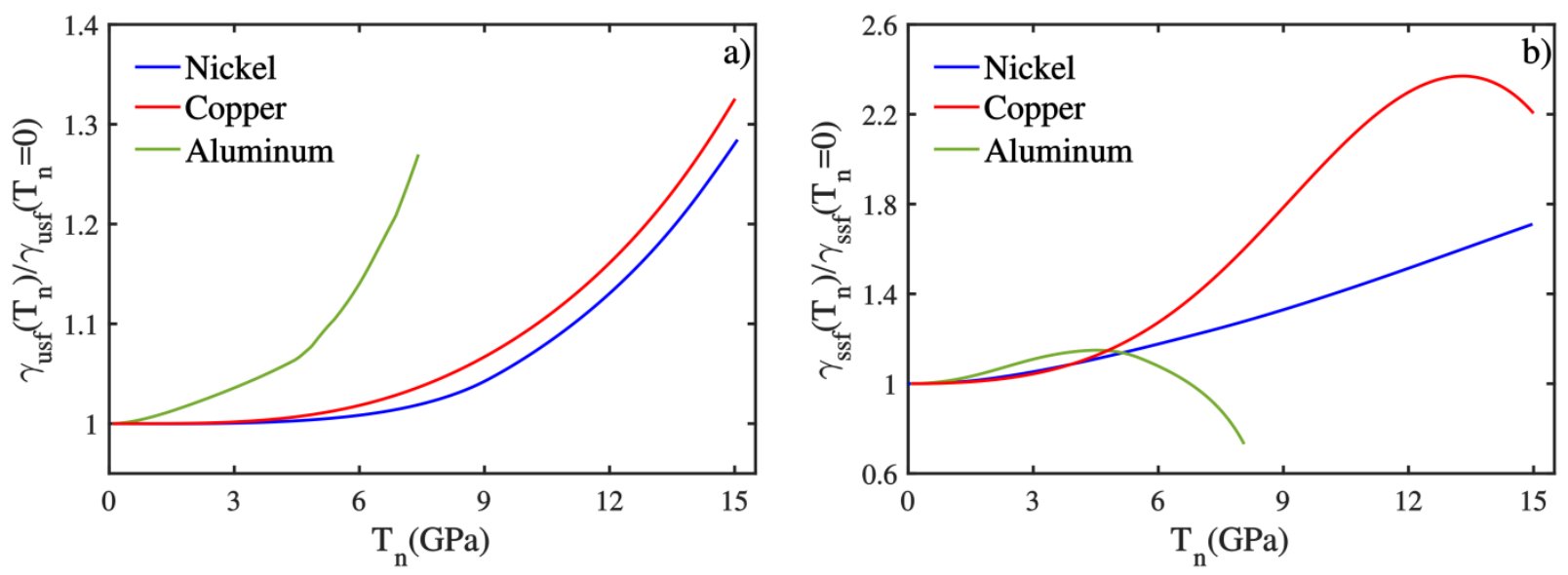

Figure 4: Normalized stable and unstable stacking fault energies in $\mathrm{Ni}$ (blue color), $\mathrm{Cu}$ (red color) and $\mathrm{Al}$ (green color) versus applied normal tensile stress.

a) $\mathrm{T}_{\mathrm{n}}=0 \mathrm{GPa}$

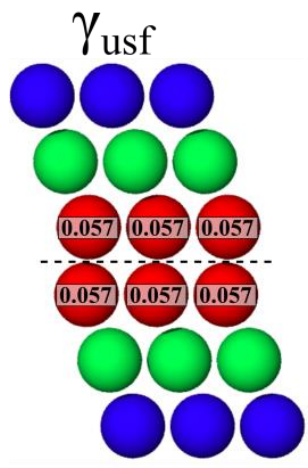

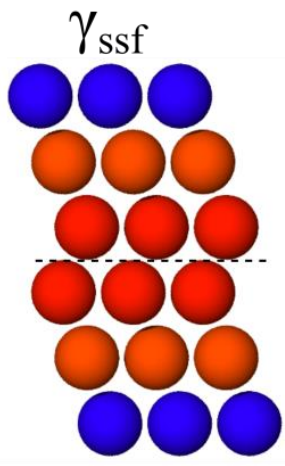

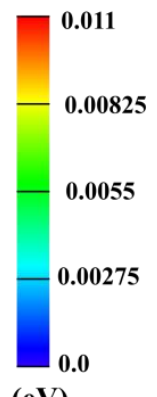

$(\mathrm{eV})$ b) $\mathrm{T}_{\mathrm{n}}=10 \mathrm{GPa}$

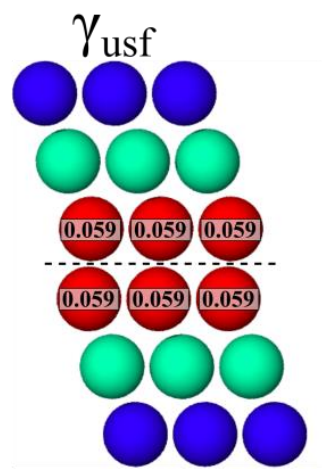

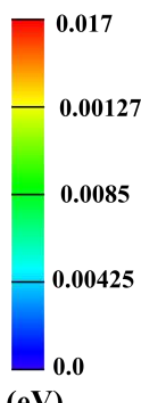

$(\mathrm{eV})$

Figure 5: Atom-by-atom energy change during rigid block shear displacement at the point of the unstable stacking energy $\gamma_{u s f}$ and stable stacking fault energy $\gamma_{s s f}$ in fcc Ni under a) zero applied stress, and b) at the applied stress $T_{n}=10 \mathrm{GPa}$. Note that contributions to the energy are confined to a few layers of atoms around the fault. Energies of atoms exceeding the energy scale are indicated.

We further examine the inelastic normal displacement under applied normal stress. The $\Delta_{n}\left(\Delta_{s}, T_{n}\right)$ represents a total change in atomic planar spacings, over several atomic layers around the stacking fault, due to the change in local atomic environment away from the perfect strained crystal. Defining $h_{i}$ as the atomic inter-planar spacing perpendicular to the slip plane between the $(i-1)^{t h}$ and $i^{\text {th }}$ planes (see Figure 2c), $\Delta_{n}\left(\Delta_{s}, T_{n}\right)$ is computed as

$$
\Delta_{n}\left(\Delta_{s}, T_{n}\right)=\sum_{i=-\infty}^{+\infty}\left[h_{i}\left(\Delta_{s}, T_{n}\right)-h_{i}\left(\Delta_{s}=0, T_{n}\right)\right]
$$

Non-zero inelastic displacements are always found over the three atomic plane spacings (central plane and one on either side of the fault $(-1 \leq i \leq 1)$ ) (Figure $2 \mathrm{c}$ ) but can extend up to five plane spacings 
This is a post-print of the following article: Andric, Predrag; Yin, Binglun; Curtin, W.A. Journal of the Mechanics and Physics of Solids 2019,, 262-279.. The formal publication is available at http://dx.doi.org/10.1016/j.jmps.2018.09.007 @ 2019. This manuscript version is made available under the CC-BY-NC-ND 4.0 license http://creativecommons.org/licenses/by-nc-nd/4.0/

$235(-2 \leq i \leq 2)$ in some cases as computed by first principles methods. We note that in DFT computations there are very small variations in interplanar spacing throughout the entire cell due to non-zero forces below the DFT tolerance level; these are numerical noise and arise even in simulations of the stressed perfect crystal. Figure 3(ii) shows the total inelastic normal displacement $\Delta_{n}\left(\Delta_{s}, T_{n}\right)$ computed via interatomic potentials for $\mathrm{Ni}, \mathrm{Cu}$, and $\mathrm{Al}$ at the various normal stresses $T_{n}$. $\Delta_{n}$ generally increases with increasing applied stress. Only in $\mathrm{Al}$ at high shear displacement and high normal stress does the opening decrease. Figure 6(ii) shows similar trends in $\Delta_{n}$ obtained from the first principle calculations, with no decreases in $\Delta_{n}$ observed in any materials. Overall, the inelastic normal displacements follow the same trends with stress as the GSFE.

From the results in Figures 3(i,ii), we can computed the GSFE $\Psi_{g s f}\left(\Delta_{S}, \Delta_{n}\right)$ for different magnitudes of the inelastic normal displacement $\Delta_{n}$ over certain ranges of displacement as shown in Figures 3(iii). Only those inelastic normal displacements corresponding to equilibrium conditions at the imposed normal stresses are accessible but the trends are clear. Figure 3(iii) clearly demonstrates an "opening hardening" trend: the GSFE increases with increasing $\Delta_{n}$. The physical origin of the "opening hardening" is not clear. At high stresses where the effect is largest, the entire material is deforming non-linearly and the inelastic normal displacements also vary with applied load. Hence, we have not been able to attribute the small total energy changes (that ultimately lead to increases in the fault energy) to a single specific mechanism.

The GSFE and the total inelastic normal displacement then allow for the computation of the generalized stacking fault enthalpy (GSFH) as

$$
\Psi_{g s f}^{\text {enth }}\left(\Delta_{s}, T_{n}\right)=\Psi_{g s f}\left(\Delta_{s}, T_{n}\right)-T_{n} \Delta_{n}\left(\Delta_{s}, T_{n}\right) .
$$

255 Figures 3(iv) and 6(iii) show the GSFH versus shear displacement $\Delta_{s}$ and normal stress $T_{n}$ for all cases studied here. The GSFH always decreases when $\mathrm{T}_{n}>0$, although $\Delta_{n}$ is not always positive, and the decreases can be a significant fraction of the zero-stress energy. Incorporating this apparent "opening softening" directly into mechanics problems as a replacement for the GSFE is, however, not quite accurate. The GSFH is measured only when the planar fault extends entirely across the area of the system. Geometry effects in mechanics problems differ, and thus the use of GSFH is not appropriate in all cases. The combination of $\Psi_{g s f}\left(\Delta_{s}, T_{n}\right)$ and $\Delta_{n}\left(\Delta_{s}, T_{n}\right)$, or the use of $\Psi_{g s f}\left(\Delta_{s}, \Delta_{n}\right)$ is more general, as we will show below. 
This is a post-print of the following article: Andric, Predrag; Yin, Binglun; Curtin, W.A. Journal of the Mechanics and Physics of Solids 2019, , 262-279.. The formal publication is available at http://dx.doi.org/10.1016/j.jmps.2018.09.007 @ 2019. This manuscript version is made available under the CC-BY-NC-ND 4.0 license http://creativecommons.org/licenses/by-nc-nd/4.0/

a) copper

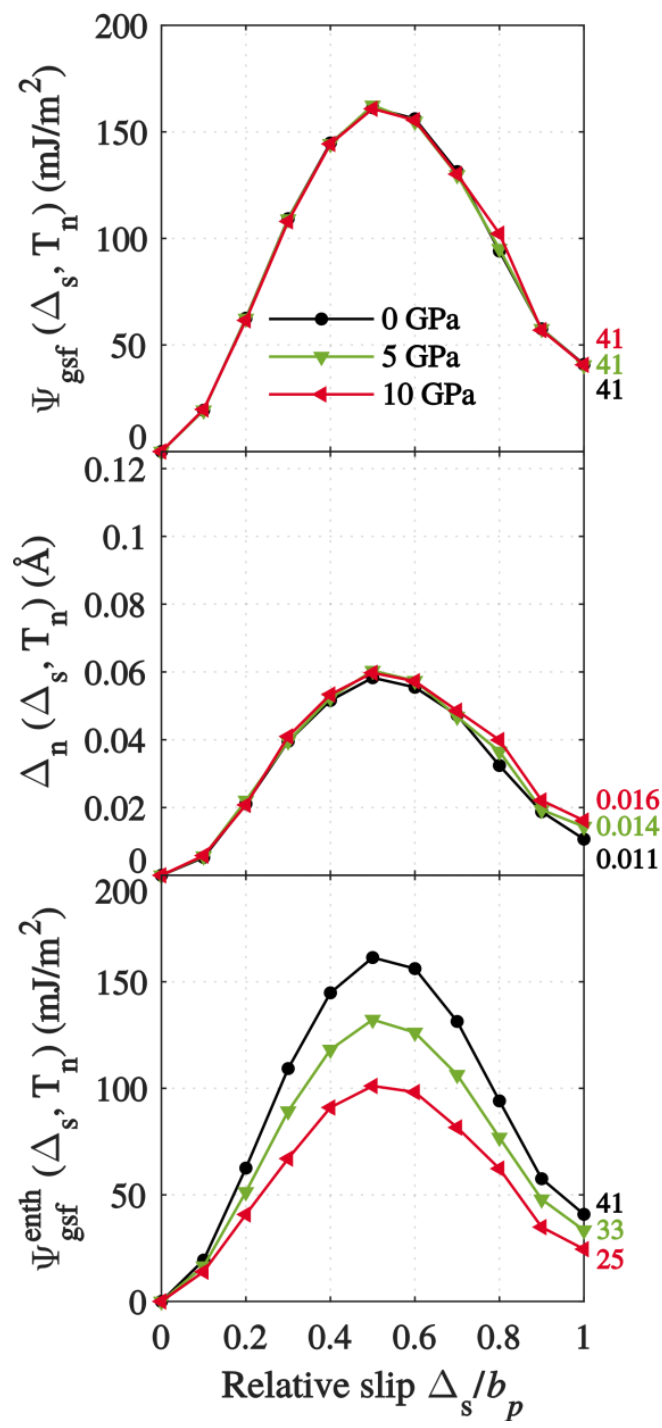

b) aluminum

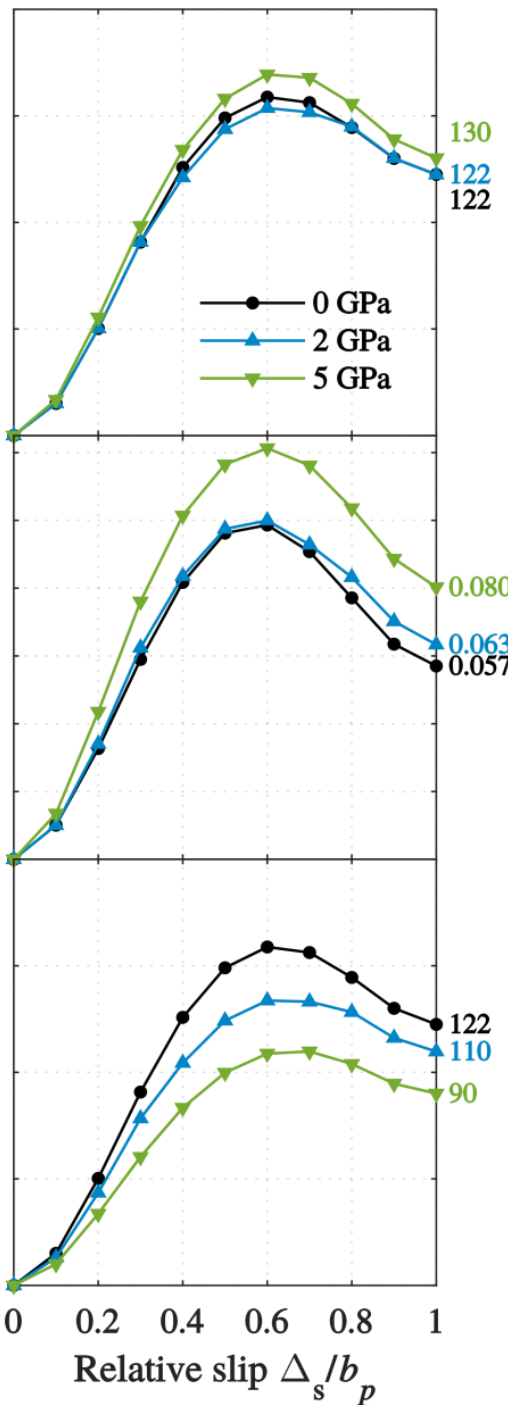

c) magnesium $\{0001\}$

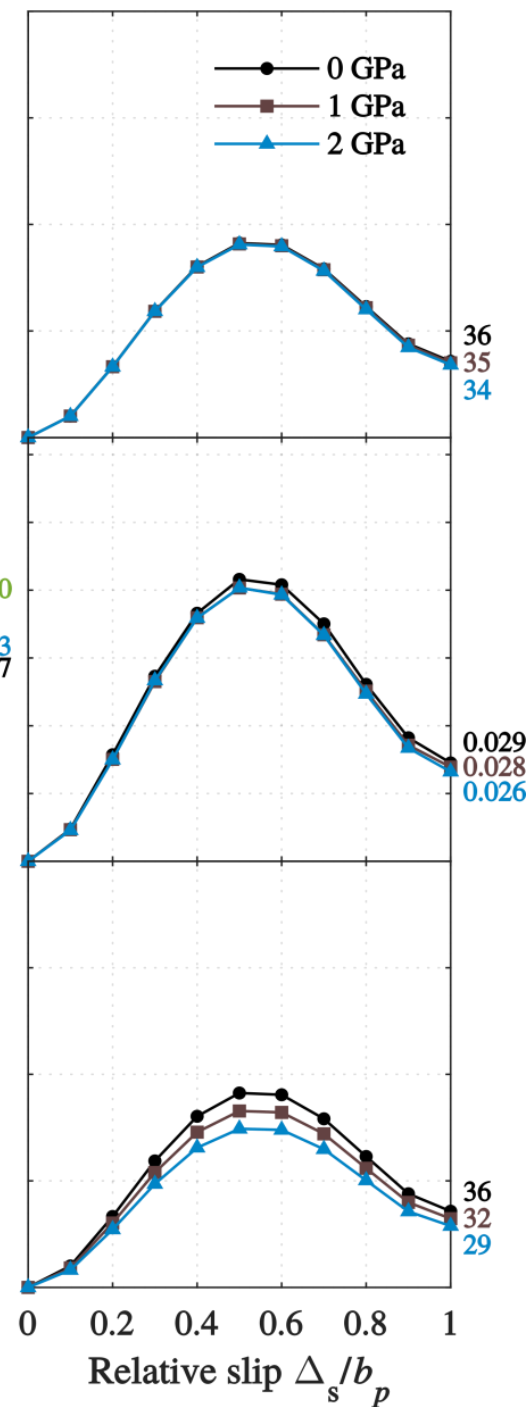

Figure 6: (i) The GSFE $\Psi_{g s f}$, (ii) total inelastic normal displacement $\Delta_{n}$ across the slip plane, and (iii) GSFH versus shear displacement $\Delta_{s}$ for different applied normal stresses as computed in DFT in a) copper, b) aluminum and c) magnesium $\{0001\}$.

\section{GSFE under normal applied loading: boundary conditions and size effects}

Direct use of stress boundary conditions leads to clear results and insights about the GSFE and the inelastic normal displacement $\Delta_{n}\left(\Delta_{s}, T_{n}\right)$, from which the GSFH can be computed. Previous computations reporting "opening softening" used displacement boundary conditions [14-17]. The enthalpy change associated with a localized planar defect in a material can be computed using either stress or displacement boundary conditions at infinity, and so in principle boundary conditions should not matter. However, only the enthalpy is obtained and simulations, especially DFT studies, are not performed on infinite systems, leading to size-dependent differences. For the stacking fault problem under an imposed normal displacement, the inelastic normal displacements lead to relaxation of the stresses throughout the entire simulation cell so as to maintain the imposed displacements on the cell. The relaxed stresses lead to relaxed elastic energies far from the stacking fault that are included into 
This is a post-print of the following article: Andric, Predrag; Yin, Binglun; Curtin, W.A. Journal of the Mechanics and Physics of Solids $2019,, 262-279$.. The formal publication is available at http://dx.doi.org/10.1016/j.jmps.2018.09.007 () 2019. This manuscript version is made available under the CC-BY-NC-ND 4.0 license http://creativecommons.org/licenses/by-nc-nd/4.0/

the calculation of the fault energy. The result is not the GSFE nor the GSFH, but asymptotically approaches the GSFH for large sizes.

The analysis of finite-sized cells under displacement control is simple because the planar fault problem is essentially one-dimensional in the $\mathrm{Y}$ dimension only. In a cell of length $L_{Y}$ with imposed total displacement $u_{y}$, the strain in the reference calculation at zero shear displacement is $\varepsilon_{y y}=u_{y} / L_{Y}$. The total stored elastic energy in the cell of volume $\mathrm{V}$ is then

$$
E_{\text {elastic }}=\frac{1}{2} C \varepsilon_{y y}^{2} V
$$

and the normal traction is $T_{n}=C \varepsilon_{y y}$, where $C$ is the plane-strain elastic modulus for loading in the $\mathrm{Y}=[11 \overline{1}]$ direction. At shear displacement $\Delta_{s}$, with inelastic normal displacement $\Delta_{n}\left(\Delta_{s}, T_{n}\right)$ across the cross-section of the cell, the total displacement is unchanged but now given by $u_{y}=\Delta_{n}+\varepsilon_{y y} L_{Y}$.

285 The elastic strain is thus decreased to $\varepsilon_{y y}-\Delta_{n}\left(\Delta_{s}, T_{n}\right) / L_{Y}$. The stored elastic energy in the system is then

$$
E_{\text {elastic }}=\frac{1}{2} C\left(\varepsilon_{y y}-\Delta_{n}\left(\Delta_{S}, T_{n}\right) / L_{Y}\right)^{2} V .
$$

The elastic energy released upon introduction of the shear displacement is the difference between Eqs. 4 and 5. Dividing by the area $A$, with $V=A L_{Y}$, leads to

$$
\frac{\Delta E_{\text {elastic }}(\Delta)}{A}=-C \varepsilon_{y y} \Delta_{n}\left(\Delta_{s}, T_{n}\right)+\frac{1}{2} C \frac{\Delta_{n}^{2}\left(\Delta_{s}, T_{n}\right)}{L_{Y}}=-T_{n} \Delta_{n}\left(\Delta_{s}, T_{n}\right)+\frac{1}{2} C \frac{\Delta_{n}^{2}\left(\Delta_{s}, T_{n}\right)}{L_{Y}} .
$$

The change in total energy, including the actual change in potential energy $\Psi_{g s f}\left(\Delta_{s}, T_{n}\right)$ of the fault, is then

$$
\begin{aligned}
\Psi_{g s f}^{\text {disp }}\left(\Delta_{s}, T_{n}\right) & =\Psi_{g s f}\left(\Delta_{s}, T_{n}\right)+\frac{\Delta E_{\text {elastic }}(\Delta)}{A} \\
& =\Psi_{g s f}\left(\Delta_{s}, T_{n}\right)-T_{n} \Delta_{n}\left(\Delta_{s}, T_{n}\right)+\frac{1}{2} C \frac{\Delta_{n}^{2}\left(\Delta_{s}, T_{n}\right)}{L_{Y}}
\end{aligned}
$$

This result is neither the GSFE nor the GSFH, although it approaches the GSFH as $L_{Y} \rightarrow \infty$. The rate of approach to the GSFH depends on $\Delta_{n}\left(\Delta_{s}, T_{n}\right)$, which is unknown in advance and is both tractiondependent and material dependent. Computations using a single simulation cell size for different tractions and/or different materials thus introduce uncontrolled errors in spite of appearing to be systematic.

We demonstrate the convergence issue explicitly using interatomic potentials under displacement boundary conditions as follows. We use same rectangular simulation cell (X $=[112]$, $\mathrm{Y}=[11 \overline{1}], \mathrm{Z}=[\overline{1} 10])$ and later cell dimensions $\left(\mathrm{X}=6 \sqrt{6} a_{0}, Z=2 \sqrt{2} a_{0}\right)$ with periodic boundary conditions in $\mathrm{X}$ and $\mathrm{Z}$. We consider Y lengths between 10 and $240\langle 111\rangle$ atomic planes. For a desired 
This is a post-print of the following article: Andric, Predrag; Yin, Binglun; Curtin, W.A. Journal of the Mechanics and Physics of Solids 2019, , 262-279.. The formal publication is available at http://dx.doi.org/10.1016/j.jmps.2018.09.007 @ 2019. This manuscript version is made available under the CC-BY-NC-ND 4.0 license http://creativecommons.org/licenses/by-nc-nd/4.0/

300 applied normal stress $T_{n}$, the corresponding applied normal strain $\varepsilon_{y y}$ is computed from Hooke's Law. The atomic positions are initialized by linearly displacing all atoms at $y$ by $y \varepsilon_{y y}$ in the [111] direction. The initial total energy $\mathrm{E}\left(\Delta_{s}=0, \varepsilon_{y y}\right)$ is computed by relaxing all the non-boundary atoms using the "fire" method [21], while the boundary atoms (atoms within $r_{c}$ of the Y boundaries at $y=$ 0 and $y=L_{y}$ ) are held fixed at the imposed displacements corresponding to the applied strain $\varepsilon_{y y}$ (see Figure 2a). We then rigidly slide the upper half of the crystal in [112] slip direction, holding the $\mathrm{Y}$ positions of the boundary atoms fixed and allowing all other atoms to relax in the $\mathrm{Y}$ direction normal to the slip plane. The total energy of the simulation cell $\mathrm{E}\left(\Delta_{S}, \varepsilon_{y y}\right)$ versus slip displacement is then computed for all three studied materials. The apparent energy change is then calculated using Eq. 1.

Figure 7 shows the normalized values of $\gamma_{u s f}$ and $\gamma_{s s f}$ at the applied strain of $\varepsilon_{y y}=0.045$ as a function of the $\mathrm{Y}$ dimension (number $\mathrm{N}$ of [11 1 ] atomic layers). This applied strain corresponds to an applied stress of $\sim 15.8 \mathrm{GPa}$ in $\mathrm{Ni}, \sim 9.8 \mathrm{GPa}$ in $\mathrm{Cu}$, and $\sim 5.1 \mathrm{GPa}$ in $\mathrm{Al}$. Since all other points on the GSFE curve follow a similar evolution we do not present them. Figure 7 shows that at least 100 [111] atomic layers are needed for reasonable converged results $\Psi_{g s f}^{\text {disp }}\left(\Delta_{s}, T_{n}\right) \sim \Psi_{g s f}^{\text {enth }}\left(\Delta_{s}, T_{n}\right)$ at this 315 applied strain. In addition, since we do not control either the normal traction nor the inelastic normal displacements, and since the material might be nonlinearly elastic at high strains, the simulation results in Figure 7 may not be corrected simply by using Eq. 7.
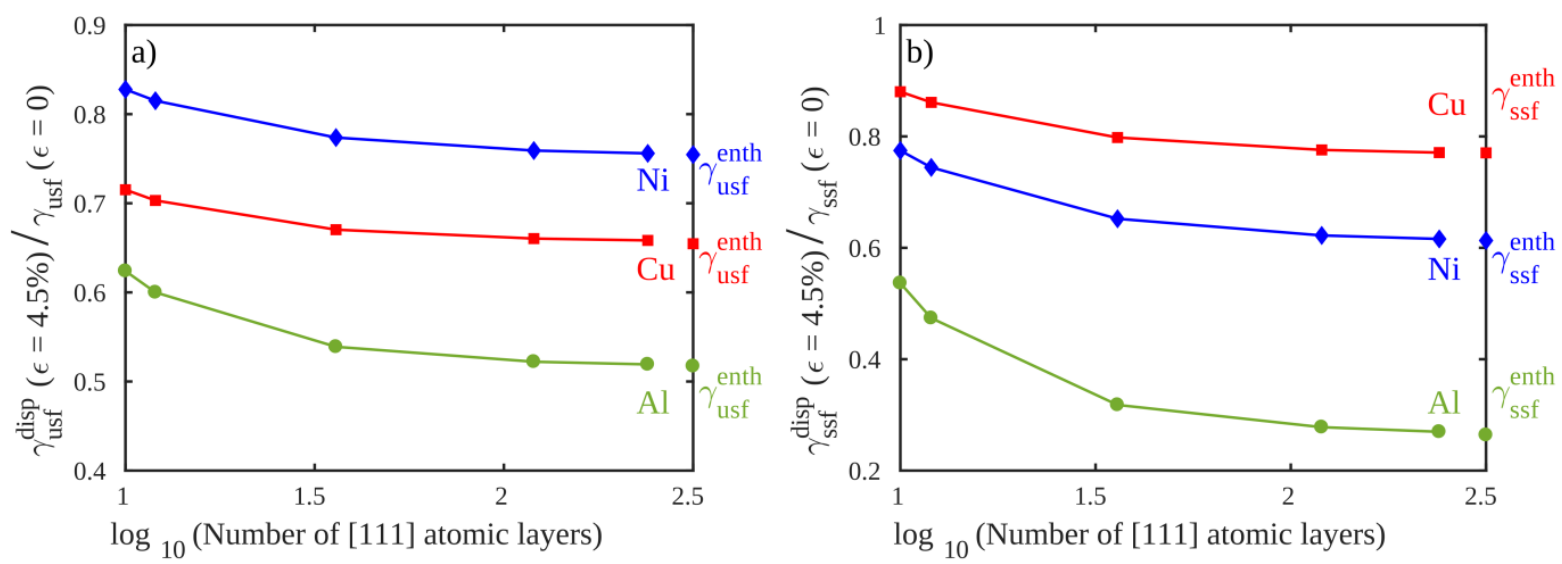

Figure 7: The size dependence of a) $\gamma_{u s f}^{\text {disp }}$ and b) $\gamma_{s s f}^{\text {disp }}$ using displacement boundary conditions along with $\gamma_{u s f}^{\text {enth }}$ and $\gamma_{s s f}^{\text {enth }}$ computed using Eq. 3 and shown on the right axis, at the applied strain of $\varepsilon_{y y}=0.045$ in nickel (blue diamonds), copper (red squares) and aluminum (green circles).

The use of displacement boundary conditions in DFT has further been studied in $\mathrm{Cu}$ for the stable stacking fault energy $\gamma_{s s f}$. The displacement boundary conditions are as described above, but using the tilted cell method and a cell length of 12 atomic layers. Table 1 shows $\gamma_{s s f}$ and $\Delta_{n}$ computed using stress boundary conditions along with $\gamma_{s s f}^{\text {enth }}$, computed using Eq. 3, and $\gamma_{s s f}^{\text {disp }}$ computed using displacement boundary conditions for different applied normal stresses. With increasing applied 
This is a post-print of the following article: Andric, Predrag; Yin, Binglun; Curtin, W.A. Journal of the Mechanics and Physics of Solids 2019, , 262-279.. The formal publication is available at http://dx.doi.org/10.1016/j.jmps.2018.09.007 (C) 2019. This manuscript version is made available under the CC-BY-NC-ND 4.0 license http://creativecommons.org/licenses/by-nc-nd/4.0/

stress, there is a difference between $\gamma_{s s f}^{\text {disp }}$ and $\gamma_{s S f}^{\text {enth }}$, with $\gamma_{s s f}^{\text {enth }}<\gamma_{s s f}^{\text {disp }}$ as expected. The difference 325 here is not large because $\Delta_{n}\left(\Delta_{s}=\mathrm{b}_{p}, \mathrm{~T}_{n}\right)$ in $\mathrm{Cu}$ is particularly small (see figure 6(ii))) but the trend is clear. Larger errors would be found in the other materials using the same cell size. Thus, obtaining converged results in DFT using displacement boundary conditions is not attractive since (i) the inelastic normal displacement $\Delta_{n}\left(\Delta_{s}, \mathrm{~T}_{n}\right)$ is not known a priori, (ii) the size needed for convergence is not clearly established a priori, and (iii) the direct application of stress on much smaller cells, as done here, provides all the necessary information with far less computational effort.

\begin{tabular}{ccccc}
\hline$T_{n}$ & $\gamma_{s s f}$ & $\Delta_{n}$ & $\gamma_{s f f}^{\text {enth }}(\mathrm{Eq} .3)$ & $\gamma_{s s f}^{\text {disp }}$ \\
$(\mathrm{GPa})$ & $\left(\mathrm{mJ} / \mathrm{m}^{2}\right)$ & $(\AA)$ & $\left(\mathrm{mJ} / \mathrm{m}^{2}\right)$ & $\left(\mathrm{mJ} / \mathrm{m}^{2}\right)$ \\
\hline 0 & 40.9 & 0.011 & 40.9 & 41.0 \\
5 & 40.6 & 0.014 & 33.6 & 34.6 \\
10 & 40.7 & 0.016 & 24.7 & 27.1 \\
\hline
\end{tabular}

Table 1: Stable stacking fault energy $\gamma_{s s f}$ and inelastic normal displacement $\Delta_{n}$ computed in copper via DFT using stress boundary conditions along with the stable stacking fault enthalpy $\gamma_{s s f}^{\text {enth }}$ computed using Eq. 3, and $\gamma_{s s f}^{\text {disp }}$ computed using displacement boundary conditions.

\section{Applications of the GSFE}

Section 3 has clearly highlighted that the GSFE and GSFH are the two thermodynamic quantities that arise during GSF calculations under an applied normal stress. In addition, the crucial feature above is the inelastic normal displacement $\Delta_{n}$ across the stacking fault plane that enters in both $\Psi_{g s f}$ and $\Psi_{g s f}^{e n t h}$. Here, we examine which thermodynamic quantity is most appropriate to use for describing different mechanics problems.

\subsection{GSFE: An analysis based on the Eshelby method}

The stacking fault induces normal inelastic displacements $\Delta_{n}$ of the atomic planes just around the fault plane (Figure $2 \mathrm{~b}$ and 3(ii)). The local nature of $\Delta_{n}$ suggests that stress dependence of the stacking fault can be derived using an Eshelby-type analysis [27] where the inelastic normal

345 displacement is treated as an "eigenstrain" $\boldsymbol{\varepsilon}^{T}$ associated with an "inclusion" confined to the stacking fault region. Any finite length stacking fault must be bounded by partial dislocations but here we first outline the general Eshelby analysis and then in Sec. 5.2 we apply it to the prediction of the fcc Shockley partial dissociation distance under applied normal stress. 
This is a post-print of the following article: Andric, Predrag; Yin, Binglun; Curtin, W.A. Journal of the Mechanics and Physics of Solids $2019,, 262-279$.. The formal publication is available at http://dx.doi.org/10.1016/j.jmps.2018.09.007 () 2019. This manuscript version is made available under the CC-BY-NC-ND 4.0 license http://creativecommons.org/licenses/by-nc-nd/4.0/

Following standard analysis, consider a body with no inclusion that is subjected to external surface tractions $\boldsymbol{T}^{A}$ applied over the boundary $\mathrm{S}$. The total free energy of the body, which is the generalized enthalpy, is

$$
F_{1}=\frac{1}{2} \int_{V} \boldsymbol{\sigma}^{A}: \varepsilon^{A} d V-\int_{S} \boldsymbol{T}^{A} \boldsymbol{u}^{A} d S
$$

where $\boldsymbol{\sigma}^{A}, \boldsymbol{\varepsilon}^{A}$ and $\boldsymbol{u}^{A}$ are the stress, strain, and displacement due to the applied load $\boldsymbol{T}^{A}$. The first term is the elastic energy stored in the volume $\mathrm{V}$ and the second term is the work done by the applied tractions $\boldsymbol{T}^{A}$ on $S$. Now consider the insertion of an inclusion of area $A$ having the same elastic moduli as the body but having a chemical energy change $\Psi_{g s f} A$ and undergoing some eigenstrain $\boldsymbol{\varepsilon}^{T}$. The enthalpy of the system is then

$$
F_{2}=\frac{1}{2} \int_{V}\left(\boldsymbol{\sigma}^{A}+\boldsymbol{\sigma}\right):\left(\boldsymbol{\varepsilon}^{A}+\boldsymbol{\varepsilon}-\boldsymbol{\varepsilon}^{T}\right) d V-\int_{S} \boldsymbol{T}^{A}\left(\boldsymbol{u}^{A}+\boldsymbol{u}\right) d S+\Psi_{g s f} A
$$

where $\boldsymbol{\sigma}, \boldsymbol{\varepsilon}-\boldsymbol{\varepsilon}^{T}$ and $\boldsymbol{u}$ are additional and unknown stress, elastic strain, and displacement fields, respectively, generated by the inclusion that undergoes some eigenstrain $\boldsymbol{\varepsilon}^{T}$. Using integration by parts, Gauss law, and some simple algebraic manipulations (for more details see [27-28]) the enthalpy can be written as

$$
F_{2}=\frac{1}{2} \int_{V} \boldsymbol{\sigma}^{A}: \boldsymbol{\varepsilon}^{A} d V-\int_{S} \boldsymbol{T}^{A} \boldsymbol{u}^{A} d S-\frac{1}{2} \int_{V} \boldsymbol{\sigma}: \boldsymbol{\varepsilon}^{T} d V-\int_{S} \boldsymbol{T}^{A} \boldsymbol{u} d S+\Psi_{g s f} A .
$$

The third and fourth terms are elastic energy stored in the body only due to the inclusion eigenstrain and an interaction energy corresponding to the external work done by the deformations caused by the inclusion, respectively. The change in enthalpy is then

$$
\Delta F=-\frac{1}{2} \int_{V} \boldsymbol{\sigma}: \boldsymbol{\varepsilon}^{T} d V-\int_{S} \boldsymbol{T}^{A} \boldsymbol{u} d S+\Psi_{g s f} A
$$

Our computational results above shows that the $\Psi_{g s f}$ itself is stress-dependent, but this can be folded into Eq. 11 by self-consistently computing the actual stress inside the inclusion. This does not change the main features of our analysis.

The analysis presented above is rather general and does not depend on the inclusion shape. Now, consider a plate-like inclusion that extends across the entire crystal. This corresponds to the stacking fault configuration used to compute the GSFH (see Sec. 2 and Figure 2). The first term in Eq. 11 then disappears since the inclusion is not constrained by the surrounding elastic material and therefore generates no additional stress $(\sigma \rightarrow 0)$. Thus, the change in crystal enthalpy becomes

$$
\Delta F=-T_{n} \Delta_{n} A+\Psi_{g s f} A
$$


This is a post-print of the following article: Andric, Predrag; Yin, Binglun; Curtin, W.A. Journal of the Mechanics and Physics of Solids 2019,, 262-279.. The formal publication is available at http://dx.doi.org/10.1016/j.jmps.2018.09.007 @ 2019. This manuscript version is made available under the CC-BY-NC-ND 4.0 license http://creativecommons.org/licenses/by-nc-nd/4.0/

where $2 \boldsymbol{u}=\Delta_{n}=$ const on the body's outer boundary $(\boldsymbol{u} / 2$ displacements on the top and bottom surfaces normal to the fault). Dividing Eq. 12 by the stacking fault area leads to

$$
\frac{\Delta F}{A}=-T_{n} \Delta_{n}+\Psi_{g s f} \equiv \Psi_{g s f}^{e n t h},
$$

which is precisely the GSFH. Note that Eq. 13 is only valid when the stacking fault region exists across the entire crystal. When the stacking fault region is confined, e.g. surrounded by elastic material (Shockley partial dislocations in fcc metals, dislocation emerging from a grain boundary, etc.), the change in crystal enthalpy is given by Eq. 11 .

Further integration of Eq. 11 using Gauss law leads to the alternative expression

$$
\frac{\Delta F}{A}=-\frac{1}{2 A} \int_{V_{I}} \boldsymbol{\sigma}: \boldsymbol{\varepsilon}^{T} d V-\frac{1}{A} \int_{V_{I}} \boldsymbol{\sigma}^{A}: \boldsymbol{\varepsilon}^{T} d V+\Psi_{g s f} .
$$

The two volume integrals are non-zero only within the inclusion volume $\mathrm{V}_{\mathrm{I}}$ because $\boldsymbol{\varepsilon}^{T}$ exists only inside the inclusion, regardless of the inclusion shape. Often, the first term is small because the additional stress caused by the inclusion eigenstrain is proportional to $\boldsymbol{\varepsilon}^{T}$ and so this term is second order in $\boldsymbol{\varepsilon}^{T}$. The second term is similar to the contribution $-T_{n} \Delta_{n}$ but is not exactly the same. From Eq. 14, it is clear that, even for an infinite elastic space subject to traction boundary conditions, the GSFH emerges only as a special case for a specific geometry (the infinite fault), although the overall free energy change has an "enthalpy-like" nature to it (due to the second term in Eq. 14).

\subsection{Shockley partial dislocation dissociation under applied normal stress}

The analysis presented in the previous section can be applied to analyze the stress dependence of fcc partial dislocation spacing. In fcc metals, it is energetically favorable for a perfect dislocation of Burgers vector $\frac{1}{2}\langle 110\rangle$ to dissociate into two Shockley partial dislocations with Burgers vectors of the $\frac{1}{6}\langle 112\rangle$ type [2]. The slip in between the two partial dislocations, along the $\langle 112\rangle$ direction between closed-packed $\{111\}$ atomic planes, generates a stacking fault with energy cost (per unit area) $\gamma_{s s f}=\Psi_{g s f}\left(\Delta=b_{p}, \boldsymbol{T}^{A}=0\right)$. The elastic interactions between the two partials are repulsive and so the partial dislocations have an equilibrium separation that balances the elastic and stacking fault energies.

At zero applied normal stress, the analysis is standard; we use isotropic elasticity to enable clear analytic expressions. We consider an infinitely long edge dislocation lying along the $\boldsymbol{\xi}=\widehat{\boldsymbol{x}}$ and dissociated into two parallel partials with a stacking fault between them (Figure 8). The partial Burgers vectors $\boldsymbol{b}_{1}$ and $\boldsymbol{b}_{2}$ lie at angles $\theta_{1}=60^{\circ}$ and $\theta_{2}=120^{\circ}$ with respect to the dislocation line 
This is a post-print of the following article: Andric, Predrag; Yin, Binglun; Curtin, W.A. Journal of the Mechanics and Physics of Solids 2019, , 262-279.. The formal publication is available at http://dx.doi.org/10.1016/j.jmps.2018.09.007 @ 2019. This manuscript version is made available under the CC-BY-NC-ND 4.0 license http://creativecommons.org/licenses/by-nc-nd/4.0/

$$
F=W_{1}+W_{2}+W_{12}+\gamma_{s s f} d
$$

where $W_{1}$ and $W_{2}$ are the elastic self-energies of the partial dislocations, and $W_{12}$ is the elastic interaction energy between the two partial dislocations [2]

$$
W_{12}=-\frac{\mu\left(\boldsymbol{b}_{1} \cdot \xi\right)\left(\boldsymbol{b}_{2} \cdot \xi\right)}{2 \pi} \ln \frac{d}{|\boldsymbol{b}|}-\frac{\mu}{2 \pi(1-v)}\left[\left(\boldsymbol{b}_{1} \times \xi\right) \cdot\left(\boldsymbol{b}_{2} \times \xi\right)\right] \ln \frac{d}{|\boldsymbol{b}|}
$$

with $\mu$ and $v$ the shear modulus for sliding along $\langle 110\rangle$ direction and Poisson's ratio, respectively. The self-energies of each partial dislocation do not depend on the partial separation and so do not influence the equilibrium separation. Therefore the equilibrium distance minimizes $W_{12}+\gamma_{s s f} d$, leading to the well-known result

$$
d=\frac{\mu b_{p}^{2}}{8 \pi \gamma_{s s f}} \frac{v+2}{1-v} .
$$

Using material properties for fcc $\mathrm{Ni}$ and $\mathrm{Al}$ as described by the EAM potential [11] (see Appendix B), the dissociation distances are $d_{0}=16.3 \AA$ and $d_{0}=10.1 \AA$, respectively. A full anisotropic analysis for Ni yields $19.1 \AA$, but anisotropy does not affect the major findings below.

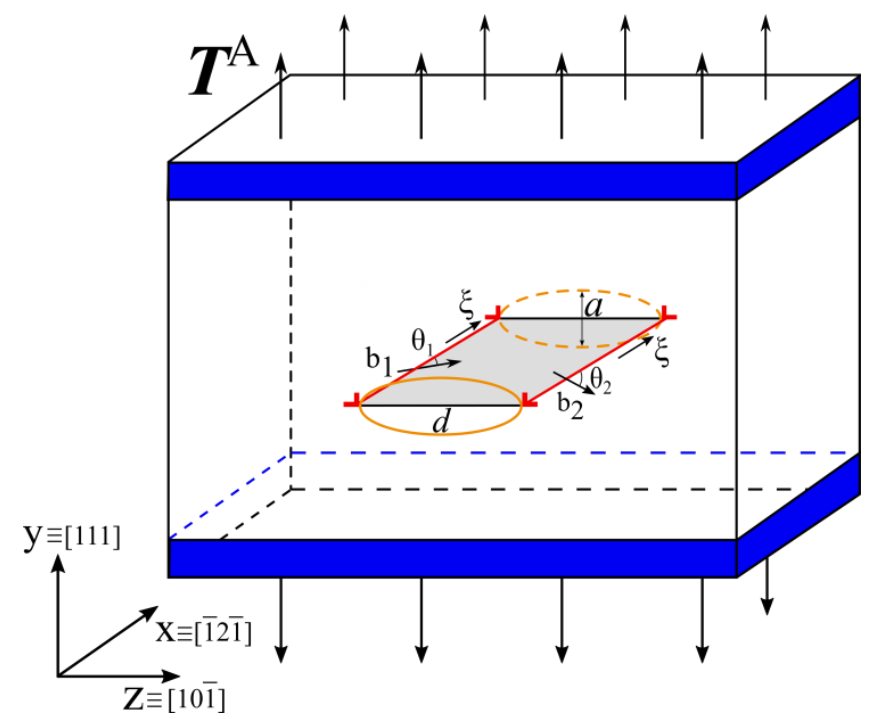

Figure 8: Geometry used to simulate the equilibrium separation $d$ between Shockley partial dislocations under normal stress applied that is perpendicular to the slip plane (along y axis). Red color indicates partial dislocations and dislocation lines along $\mathrm{x}$ axis, while the grey color represents the stacking fault. Orange color indicates the elliptic cylinder that undergoes some eigenstrain $\varepsilon^{T}=\varepsilon_{y y}^{T}=\Delta_{n}\left(\Delta_{s}, T_{n}\right) / a$. Blue color indicates boundary atoms.

We now examine the partial dislocation dissociation distance with increasing applied stress perpendicular to the slip plane $\boldsymbol{T}^{A}=T_{y}=T_{n}$. Eq. 17 suggests that applied normal stress nominally affects the stable stacking fault energy. Because the stacking fault defect is completely surrounded by linearly elastic material, we treat the stacking fault as a defect with an eigenstrain using the analysis 415 of the previous section (Eq. 14), as follows. The inelastic normal displacements exist over several atomic planes around the fault plane. We thus consider the stacking fault as an elliptic cylindrical inclusion having major axis length $d$ (the stacking fault length) and minor axis length $a=3 h$ where 
This is a post-print of the following article: Andric, Predrag; Yin, Binglun; Curtin, W.A. Journal of the Mechanics and Physics of Solids 2019, , 262-279.. The formal publication is available at http://dx.doi.org/10.1016/j.jmps.2018.09.007 @ 2019. This manuscript version is made available under the CC-BY-NC-ND 4.0 license http://creativecommons.org/licenses/by-nc-nd/4.0/

$h$ is the $\{111\}$ atomic plane spacing (Figure 8). The choice of $a=3 h$ is based on the measured inelastic normal displacements in each plane, as shown in Figure 9. The total inelastic displacement $\Delta_{n}\left(\Delta_{s}, T_{n}\right)$ is the sum over the planes. DFT calculations show inelastic displacements over one additional pair of layers, suggesting $a=5 h$, but this does not influence the main result (see below). The inclusion has the same elastic properties as the matrix and an eigenstrain $\boldsymbol{\varepsilon}^{T}=\varepsilon_{y y}^{T}=$ $\Delta_{n}\left(\Delta_{s}=b_{p}, T_{n}\right) / a$. The eigenstrain itself depends on the applied normal stress and so is a non-linear eigenstrain (see Figure 9); again, this does not influence the general analysis.
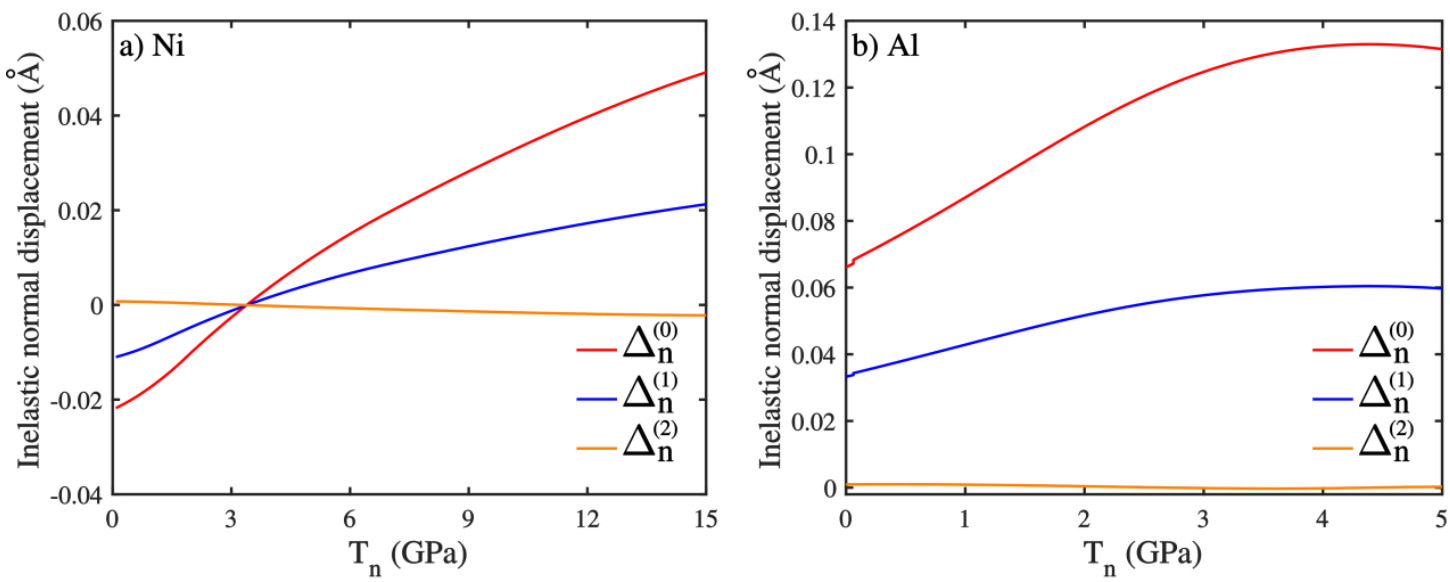

Figure 9: Inelastic vertical displacement at the stable stacking fault position $\left(\Delta_{s}=b_{p}\right)$ in a) $\mathrm{Ni}$ and b) $\mathrm{Al}$ between atomic planes immediately across the slip plane $\Delta_{n}^{(0)}$ (red color), and between atomic planes just above and below the slip plane $\Delta_{n}^{(1)}$ (blue color). The inelastic vertical displacement is zero everywhere else, as shown here for $\Delta_{n}^{(2)}$ (orange color).

Due to the inclusion with eigenstrain but with no applied stress, there is an additional elastic strain energy contribution per unit length of dislocation of

$$
W_{I}=-\frac{1}{2} \int_{A_{I}} \boldsymbol{\sigma}: \boldsymbol{\varepsilon}^{T} d A
$$

where $A_{I}=a d \pi / 4$ is area of the inclusion in the $\mathrm{y}-\mathrm{z}$ plane. The stress $\boldsymbol{\sigma}$ inside the elliptical inclusion is constant [27], and given by

$\boldsymbol{\sigma}=\boldsymbol{C}(\boldsymbol{S}-\boldsymbol{I}) \boldsymbol{\varepsilon}^{T}$

where $\boldsymbol{C}$ is the stiffness tensor, $\boldsymbol{S}$ the dimensionless Eshelby tensor for an elliptic cylinder [27, 28] and $I$ the identity tensor. Within isotropic elasticity, $C_{11}=2 \mu(1-v) /(1-2 v), C_{12}=2 \mu v /(1-$ $2 v$ ) and $C_{44}=\mu$. The elastic energy (per dislocation length) due to $\varepsilon^{T}$ is then simply

$$
W_{I}=-\frac{1}{2} \boldsymbol{C}(\boldsymbol{S}-\boldsymbol{I}) \boldsymbol{\varepsilon}^{T}: \boldsymbol{\varepsilon}^{T} A_{I}
$$

This contribution is, however, generally negligible. Note also that the interaction energy between the partial dislocations and the inclusion stress field is zero, in isotropic elasticity, because the eigenstrain $\boldsymbol{\varepsilon}^{T}$ has only one non-zero component along y direction and the dislocation stress field is antisymmetric 
This is a post-print of the following article: Andric, Predrag; Yin, Binglun; Curtin, W.A. Journal of the Mechanics and Physics of Solids $2019,, 262-279$.. The formal publication is available at http://dx.doi.org/10.1016/j.jmps.2018.09.007 () 2019. This manuscript version is made available under the CC-BY-NC-ND 4.0 license http://creativecommons.org/licenses/by-nc-nd/4.0/

with respect to the $\mathrm{x}-\mathrm{z}$ plane. So, at zero applied traction, the energy remains essentially that of Eq. 15 and the dissociation distance that of Eq. 17.

When a stress is applied normal to the stacking fault plane, the second energy contribution in Eq. 14 must be added to the total energy. For the (elliptical) inclusion with the $\boldsymbol{\varepsilon}^{\boldsymbol{T}}$, this interaction energy is

$$
W_{\sigma}=-\boldsymbol{\sigma}^{A}: \varepsilon^{\boldsymbol{T}} A_{I}
$$

The equilibrium dissociation distance $d$ then minimizes the energy

$$
W_{\text {total }}=W_{12}+\Psi_{g s f}\left(\Delta_{s}=b_{p}, T_{n}\right) d+W_{I}+W_{\sigma} .
$$

Since $\boldsymbol{\sigma}^{A}=\sigma_{y y}^{A}=T_{n}, \boldsymbol{\varepsilon}^{\boldsymbol{T}}=\varepsilon_{y y}^{T}=\Delta_{n}\left(\Delta_{s}=b_{p}, T_{n}\right) / a, A_{I}=a d \pi / 4$, and $W_{I}$ is negligible, the energy functional reduces to the simple form

$$
W=W_{12}+d\left[\Psi_{g s f}\left(\Delta_{s}=b_{p}, T_{n}\right)-\frac{\pi}{4} T_{n} \Delta_{n}\left(\Delta_{s}=b_{p}, T_{n}\right)\right] .
$$

445 The stress dependence of the GSFE thus appears to reduce the relevant stacking fault energy. The partial separation is then predicted to be

$$
d\left(T_{n}\right)=\frac{\mu b^{2}}{8 \pi\left[\Psi_{g s f}\left(\Delta_{s}=b_{p}, T_{n}\right)-\left(\frac{\pi}{4}\right) T_{n} \Delta_{n}\left(\Delta_{s}=b_{p}, T_{n}\right)\right]} \frac{v+2}{1-v} .
$$

There are four contributions to the stress dependence of $d\left(T_{n}\right)$ : (i) the interaction energy between two partials, (ii) the change of $\Psi_{g s f}\left(\Delta_{s}=b_{p}, T_{n}\right)$ with the applied stress (Figure 4), (iii) the coupling of applied stress and inelastic displacement $\Delta_{n}$, and (iv) the geometrical parameter $\pi / 4$ arising because the inelastic displacement is spread over several planes and localized to the dislocation volume, represented by the elliptical geometry. The net result is an increase in the partial dislocation separation with increasing applied normal stress - that is, there is an "opening softening". However, the direct application of the GSFH as a replacement for the GSFE would not have the factor of $\pi / 4$, and thus would predict a larger partial spacing.

As seen in Figure 9, the inelastic normal displacements at small $T_{n}$ for Ni are negative and are zero at just above $T_{n}=3 \mathrm{GPa}$. Thus, the theory predicts that the dissociation distance will actually decrease for $T_{n}<3 \mathrm{GPa}$. In Al, $\Delta_{n}$ is positive for all $T_{n}$ and much larger than in $\mathrm{Ni}$, and so the dissociation distance is predicted to increase, and more rapidly than in Ni. A subtlety in applying the elasticity analysis at high $T_{n}$ is that the underlying material becomes nonlinearly elastic. To account 460 for this, we use the tangent modulus $\mu=\mu\left(T_{n}\right)$ and Poisson ratio at the far-field applied stress $T_{n}$ (see Appendix B). This approximation is valid because the problem can be envisioned as first homogeneously deforming the entire material (non-linearly) and then adding a dissociated dislocation into this material. Linearizing around the homogeneously-deformed state enables application of superposition upon addition of the dislocation, similar to the usual introduction of a dislocation into 
This is a post-print of the following article: Andric, Predrag; Yin, Binglun; Curtin, W.A. Journal of the Mechanics and Physics of Solids 2019, , 262-279.. The formal publication is available at http://dx.doi.org/10.1016/j.jmps.2018.09.007 @ 2019. This manuscript version is made available under the CC-BY-NC-ND 4.0 license http://creativecommons.org/licenses/by-nc-nd/4.0/

an unstrained (but still non-linear) atomistic material. Figure 10 shows the partial spacing versus applied normal stress as predicted using (i) Eq. 23, and (ii) Eq. 17 with $\Psi_{s s f}^{\text {enth }}\left(\Delta_{s}=b_{p}, T_{n}\right)$. In both cases we use $\mu=\mu\left(T_{n}\right)$. There are significant quantitative differences between two cases, especially at higher applied loads. Both cases (i) and (ii) do show an "opening softening" effect, although it is almost zero in Ni for $T_{n}<3 \mathrm{GPa}$ due to the small negative inelastic eigenstrains.
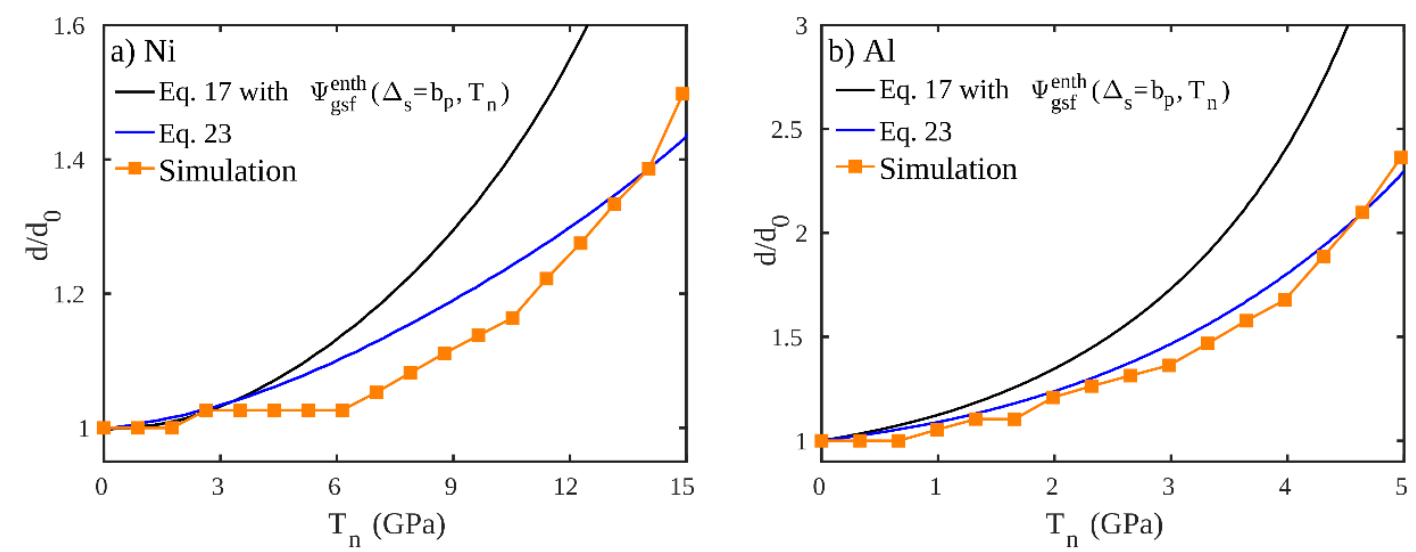

Figure 10: Equilibrium separation between partial dislocations in a) $\mathrm{Ni}$ and b) $\mathrm{Al}$ at different applied stress that is perpendicular to the slip plane, normalized with its value at zero applied load, as predicted by equation 17 with $\gamma_{S S f}^{\text {enth }}$ (black line), or by Eq. 23 (blue color), and as observed in atomistic simulations (orange squares).

To evaluate the above predictions, we execute simulations using interatomic potentials for $\mathrm{Ni}$ and Al. We use the standard methods [29], as follows. The simulation cell is oriented with $\mathrm{X}=[\overline{1} 2 \overline{1}]$, $\mathrm{Y}=[111]$ and $\mathrm{Z}=[10 \overline{1}]$ (Figure 8) with dimensions of $\sim 600 \times 25 \times 250 \AA$ (345,400 atoms) that we have verified to be sufficient for converged results. We insert a $\{111\}\langle 110\rangle$ edge dislocation by adding a periodic $\{110\}$ plane in the upper half of the crystal that is spread over predicted equilibrium separation distance at $T_{n}=0$. Periodic boundary conditions are applied along X and Z directions with free surface in Y. The system energy is then minimized by the "fire" method with force tolerance $10^{-6} \mathrm{eV} / \AA$ on every atom. The equilibrium distances are then measured as $d_{0}=21.8 \AA$ in $\mathrm{Ni}$, and $d_{0}=13.6 \AA$ in $\mathrm{Al}$; these are slightly higher than predicted by Eq. 17 but typical for fcc metals due to the neglect of non-linear effects, spreading of the partial Burgers vector, and the assumption of isotropic elasticity. A normal traction $T_{n}$ is then applied on the system via vertical forces applied on the boundary atoms within $r_{c}$ of the $\mathrm{Y}$ surface boundaries (Figure 8). The system energy is again minimized and the equilibrium partial separation measured. The simulated partial spacing versus applied stress $T_{n}$ is shown in Figure 10 along with the previous predictions. The simulations agree very well with the predictions of Eq. 23, which is the complete theory including the multi-plane distribution of inelastic normal displacements represented as an elliptical inclusion. The prediction using simply the enthalpy $\Psi_{s s f}^{\text {enth }}\left(\Delta_{s}=b_{p}, T_{n}\right)$ shows the same trend, but is much larger than observed in both $\mathrm{Ni}$ and $\mathrm{Al}$. The simulation results thus confirm the full theory. There is an "opening softening" 
This is a post-print of the following article: Andric, Predrag; Yin, Binglun; Curtin, W.A. Journal of the Mechanics and Physics of Solids $2019,, 262-279$.. The formal publication is available at http://dx.doi.org/10.1016/j.jmps.2018.09.007 @ 2019. This manuscript version is made available under the CC-BY-NC-ND 4.0 license http://creativecommons.org/licenses/by-nc-nd/4.0/

effect due to the coupling of the inelastic normal displacement to the applied stress, but it is smaller than predicted simply by using the GSFH.

\subsection{Crack-tip dislocation emission under mixed Mode II/I loading}

The previous section has shown a softening effect with applied normal stress for an existing dislocation with existing stacking fault in an infinite elastic medium. Many problems of interest involve dislocation nucleation in which the fault is emerging from some stress concentration and the relevant quantity is the unstable stacking fault energy. Here, we examine the effects of normal stress on one standard problem: emission of a (partial) dislocation from the crack tip of an fcc metal. We use Mode II loading, with an additional Mode I loading to create the normal stress acting on the plane of the dislocation nucleation. This problem cannot be treated using the Eshelby analysis of Sec. 5.1 since the partial stacking fault emerges from a crack and so is not surrounded by an infinite elastic medium.

A theory for dislocation emission from a crack tip under Mode II loading was developed by Rice [3]. In Mode II loading, the slip plane is coplanar with the crack plane (Figure 11a) and the critical Mode II stress intensity factor for dislocation emission $K_{I I e}$ depends on the unstable stacking fault energy $\gamma_{u s f}=\Psi_{g s f}\left(\Delta_{s} \approx b_{p} / 2\right)$ and the material elastic properties. The Rice theory agrees well with detailed simulations for this loading scenario [4]. For the same crack geometry under mixed Mode II/I loading, there is no exact solution. However, a numerical solution can be obtain by introducing the coupled opening-shear constitutive law $\Psi_{g s f}\left(\Delta_{S}, \Delta_{n}\right)$ along the slip plane and solving coupled integral equations for the slip displacement of an incipient dislocation emerging from the crack tip [10]. Within this framework, Rice and co-workers showed that $K_{I I e}$ decreases with increasing applied Mode I stress intensity factor $K_{I}$. However, the inelastic potential used in that work, which is derived from $\Psi_{g s f}\left(\Delta_{s}, \Delta_{n}\right)$, had explicit "opening softening" of $\gamma_{u s f}$; the predicted softening was thus not due directly to the enthalpy $\Psi_{u s f}^{\text {enth }}$. The full mechanics analysis introduces the coupling of crack-tip stresses and slip along the slip plane through the potential energy $\Psi_{g s f}\left(\Delta_{s}, \Delta_{n}\right)$.

515 In contrast to the law developed by Rice, the detailed results here show that $\gamma_{u s f}=\Psi_{g s f}\left(\Delta_{s} \approx\right.$ $\frac{b_{p}}{2}, \Delta_{n}$ ) is nearly independent of the normal opening (see Figure 3(iii)), indicating that $K_{I I e}$ should be nearly independent of an applied $K_{I}$.

To resolve the discrepancy between results in [10] and results in Figure 3(iii), and their presumed consequences on dislocation emission, we perform standard "K-test" simulations using the interatomic potentials for $\mathrm{Ni}, \mathrm{Cu}$ and $\mathrm{Al}$ as follows. We model a semi-infinite crack under planestrain stress intensity $K_{I I}+K_{I}$ loading conditions. A single fcc crystal is oriented with $X=$ [112], $Y=[11 \overline{1}]$ and $Z=[\overline{1} 10]$ and dimensions approximately $120 \times 120 \times 1 \mathrm{~nm}$ with periodic boundary 
This is a post-print of the following article: Andric, Predrag; Yin, Binglun; Curtin, W.A. Journal of the Mechanics and Physics of Solids 2019, , 262-279.. The formal publication is available at http://dx.doi.org/10.1016/j.jmps.2018.09.007 (C) 2019. This manuscript version is made available under the CC-BY-NC-ND 4.0 license http://creativecommons.org/licenses/by-nc-nd/4.0/

conditions along $\mathrm{Z}$. A crack is inserted into the material for $\mathrm{X}<0, \mathrm{Y}=0$ with the crack front along $\mathrm{Z}$ at $\mathrm{X}=0$. The cracked specimen is loaded by applying atomic displacements to all atoms corresponding 525 to the desired mixed Mode II/I anisotropic displacement field for linear elastic fracture mechanic. The entire system is then relaxed to a minimum energy while holding the boundary atoms within $2 r_{c}$ $\left(r_{c}=\right.$ cut-off distance of the interatomic potential) of the outer boundary fixed at the elastic solution. Additional load increments $\Delta K$ are applied in the same manner. More details can be found in Ref. [4]. In each simulation we hold the applied $K_{I}$ fixed at the desired value and incrementally increase the applied $K_{I I}$ until dislocation emission occurs.

Simulating an atomically sharp crack at loads below the stress intensity for Griffith cleavage $K_{I c}$ is always challenging since the crack is unstable to closure - the traction free crack surfaces cannot be simple imposed on atoms. To address this issue we remove one layer of atoms and artificially delete the atomic interactions across the newly created crack surfaces (see Figure 11).

535 Within this crack geometry, emission remains controlled only by the shear response along the slip plane, and no fictitious effects arise near the crack tip that would unduly influence the emission under mixed Mode II/I loading.

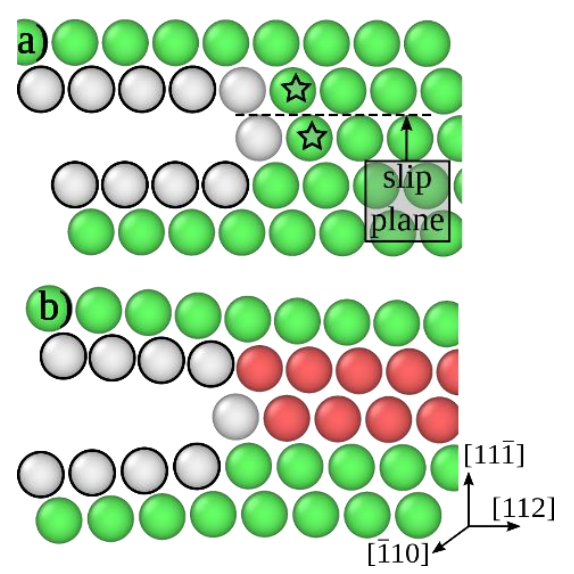

Figure 11: a) Crack geometry in mixed Mode II/I loading formed by removing one layer of atoms and then by artificially canceling the interaction between the atoms marked with black circles. b) Crack geometry after dislocation emission. Opening stress just before the emission is measured between atoms marked with stars. Atoms are colored based on Common Neighbor Analysis [30]; green for fcc, red for hcp and white for surface atoms.

Figure 12 shows the simulated $K_{I I e}$ as a function of the applied $K_{I}$ in $\mathrm{Ni}, \mathrm{Cu}$ and $\mathrm{Al}$, 540 respectively. There is no "opening softening" observed: the value of $K_{I I e}$ is essential independent of $K_{I}$. If there is any effect at all, it is a tendency toward increasing $K_{I I e}$. The near-crack-tip opening stresses (normal stresses across the slip plane), computed using the average virial stresses on the atoms marked by stars in Figure 11a, are shown in Figure 12. Due to surface effects, the atomic virial stresses on the crack tip atoms are not reliable; the stresses shown in Figure 12 are thus a lower bound. At $K_{I}=0 \mathrm{MPa} \sqrt{\mathrm{m}}$, the near-tip atoms are in normal compression. This is entirely expected because the inelastic normal displacement $\Delta_{n}$ is positive at $\Delta_{s} \approx b_{p} / 2$. At zero normal stress, the stacking fault 
This is a post-print of the following article: Andric, Predrag; Yin, Binglun; Curtin, W.A. Journal of the Mechanics and Physics of Solids 2019,, 262-279.. The formal publication is available at http://dx.doi.org/10.1016/j.jmps.2018.09.007 @ 2019. This manuscript version is made available under the CC-BY-NC-ND 4.0 license http://creativecommons.org/licenses/by-nc-nd/4.0/

cannot expand by $\Delta_{n}$ because of the constraint of the surrounding elastic material. The crack-tip inelastic displacement is then smaller than $\Delta_{n}$ and the normal stress is naturally compressive. With increasing $K_{I}$, the near-tip atoms do experience tensile opening stresses (Figure 12), as expected, and these stresses can reach levels of 5-10 GPa. Yet, $K_{I I e}$ remains unaffected by these high normal stresses. This is not surprising since (i) the local unstable stacking fault energy $\Psi_{g s f}\left(\Delta_{s} \approx b_{p} / 2, T_{n}\right)$ is constant or increasing (see Figure 4a), and (ii) the work done by $T_{n}$ over the actual crack-tip inelastic displacement is small/negligible because the crack-tip inelastic displacement is much smaller than $\Delta_{n}$. Use of the GSFH at the tip would be incorrect because it would include work done by $T_{n}$ over the $\Delta_{n}$. We conclude that proper analysis requires the use of $\Psi_{g s f}\left(\Delta_{s}, \Delta_{n}\right)$ that is derived from $\Psi_{g s f}\left(\Delta_{s}, T_{n}\right)$ and $\Delta_{n}\left(\Delta_{s}, T_{n}\right)$.

Finally, in all three materials, there is a change in the emission plane from the upper (initial) plane to the 1 atomic layer lower plane, beyond some high level of $K_{I}$. This change occurs at very high normal opening stresses, suggesting that the opening stresses on the original slip plane are suppressing emission. The emission then shifts to the lower slip plane. We cannot clearly attribute this shift to normal stress effects - there are likely additional non-linear crack-tip processes that develop, and detailed analysis is far beyond the scope of this paper. We only note that the normal stresses on the original slip plane are very high and yet emission on that plane does not occur.

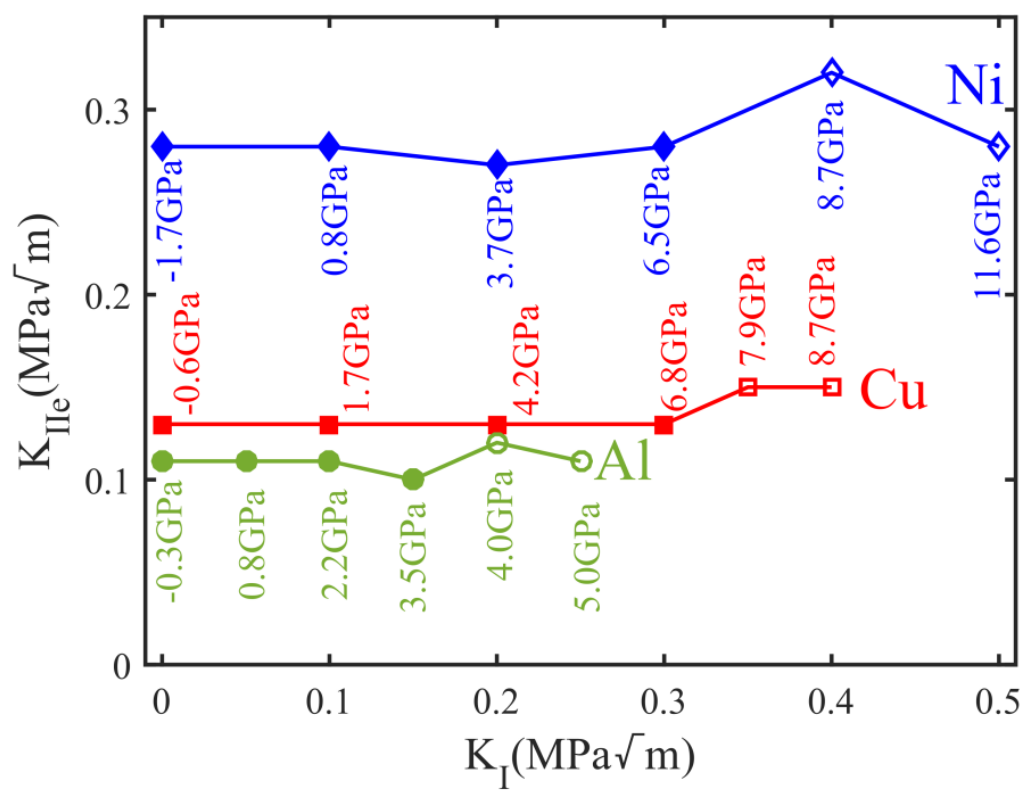

Figure 12: Molecular statics results of the critical stress intensity factor $K_{I I e}$ as a function of the applied $K_{I}$ in mixed Mode II/I loading in nickel (blue diamonds), copper (red squares) and aluminum (green circles). Cracktip opening stresses just before the emission are indicated for every applied $K_{I}$. Solid symbols correspond to the emission along the upper (initial) slip plane, while the open symbols correspond to the emission along the lower slip plane.

To summarize, no "opening softening" effect is observed in the very well controlled mixedmode $K_{I I} / K_{I}$ loading test. This is entirely consistent with the absence of "opening softening" in the 
This is a post-print of the following article: Andric, Predrag; Yin, Binglun; Curtin, W.A. Journal of the Mechanics and Physics of Solids $2019,, 262-279$.. The formal publication is available at http://dx.doi.org/10.1016/j.jmps.2018.09.007 @ 2019. This manuscript version is made available under the CC-BY-NC-ND 4.0 license http://creativecommons.org/licenses/by-nc-nd/4.0/

GSFE measured for these same materials. While the GSFH is the relevant thermodynamic quantity for specific GSF calculations under an applied stress, it is not directly relevant to the crack tip problem. Based on our examinations, reasons for the reported decrease in $K_{I I e}$ with increased $K_{I}$ in simulations reported in [10] is not due to GSFH but rather due to (i) poorly controlled crack-tip conditions in the simulations leading to spurious effects on emission, (ii) use of a $\Psi_{g s f}\left(\Delta_{s}, \Delta_{n}\right)$ computed without accounting for the non-linear response of the atomic system, and (iii) subtraction of tension elastic effects assuming only linear response of the system. This test case and the analysis in Sections 5.1 and 5.2 indicated that mechanics problems are best analyzed using the combination of $\Psi_{g s f}\left(\Delta_{s}, T_{n}\right)$ and $\Delta_{n}\left(\Delta_{s}, T_{n}\right)$ or using $\Psi_{g s f}\left(\Delta_{s}, \Delta_{n}\right)$, if computable.

\section{Concluding remarks}

Using both interatomic potentials and first principle calculations, we have demonstrated that the generalized stacking fault energy (GSFE) in various metals $(\mathrm{Ni}, \mathrm{Cu}, \mathrm{Al}, \mathrm{Mg}$ ) generally increases with increasing far-field applied tensile stress normal to the slip plane. There is almost no evidence of "opening softening" that has been envisioned and reported in the literature. An important additional outcome is that the stacking fault is not a pure-shear defect: shear sliding gives rise to stress-dependent inelastic normal displacements between the atomic planes around the slip plane, leading to a net displacement $\Delta_{n}$. This net inelastic displacement is typically positive, and does couple to the applied stress, giving rise to a generalized stacking fault enthalpy (GSFH) that does decrease with increasing normal stress. However, the apparent "opening softening" observed in the GSFH does not directly translate to "opening softening" in all mechanics problems involving stacking faults.

The above results are examined via simulation for two canonical problems: the partial dissociation spacing of dislocations in fcc metals and the emission of a dislocation from a crack tip. Theory using the GSFE and opening $\Delta_{n}$ as an eigenstrain shows that the partial spacing does increase with increasing normal stress, consistent with "opening softening", but not to the degree predicted using the GSFH. Simulations fully support the analysis. Simulations of crack tip emission under Mode II/Mode I loading show no change in the critical Mode II stress intensity for emission due to applied Mode I, and thus no "opening softening", in spite of normal stresses at the crack tip reaching 5-10 GPa. This is consistent with the need to analyze mechanics problems using the GSFE and $\Delta_{n}$ rather than the GSFH directly. In previous work on crack tip emission in Mode I, we also obtained quantitative agreement with many simulations using an analysis that does not involve any effects of normal stress on the slip behavior [4].

Overall, the present study provides a fairly complete understanding of the effects of normal stress on stacking fault energies, and demonstrates the consequences for several different situations. "Opening softening" is not a general phenomenon. Analysis of any problem requires consideration 
This is a post-print of the following article: Andric, Predrag; Yin, Binglun; Curtin, W.A. Journal of the Mechanics and Physics of Solids 2019,, 262-279.. The formal publication is available at http://dx.doi.org/10.1016/j.jmps.2018.09.007 @ 2019. This manuscript version is made available under the CC-BY-NC-ND 4.0 license http://creativecommons.org/licenses/by-nc-nd/4.0/

of the GSFE and the net inelastic opening displacement $\Delta_{n}$, and conclusions depend on the specific problem. The results and understanding here thus provide a basis for detailed investigation, or reinvestigation, of nanoscale dislocation plasticity phenomena under stresses normal to the slip plane of the dislocations.

Acknowledgements: PA and WAC acknowledge support for their work by the European Research Council through the Advanced Grant "Predictive Computational Metallurgy", ERC Grant agreement No.339081 - PreCoMet. BY acknowledges support for his work by the NCCR MARVEL.

\section{Appendix A. GSFE under uniaxial tension}

In Section 2 we investigate the GSFE stress dependence whit the lateral cell sizes held fixed. This loading scenario corresponds to the so-called uniaxial strain (imposed with the stress boundary conditions). We now investigate the influence of the lateral stresses on the GSFE by performing an additional set of GSF simulations, in which the relaxation of the lateral stresses is allowed (uniaxial tension). All other simulation details are identical to those presented in Section 2. Figure 13 shows the GSFE curves computed using interatomic potentials for $\mathrm{Ni}, \mathrm{Cu}$ and $\mathrm{Al}$ at different applied normal stresses imposed by the uniaxial strain (solid line) or uniaxial stress (dashed line). We find that lateral loads in $\mathrm{Ni}$ and $\mathrm{Cu}$ have no significant influence on the GSFE. Furthermore, the GSFE in Al tends to be more sensitive for $\Delta_{s}>0.6 b_{p}$ and for applied normal stress above $5 \mathrm{GPa}$. The observed behavior is mainly due to potential which under uniaxial tension becomes unstable for applied stresses slightly above $6 \mathrm{GPa}$.
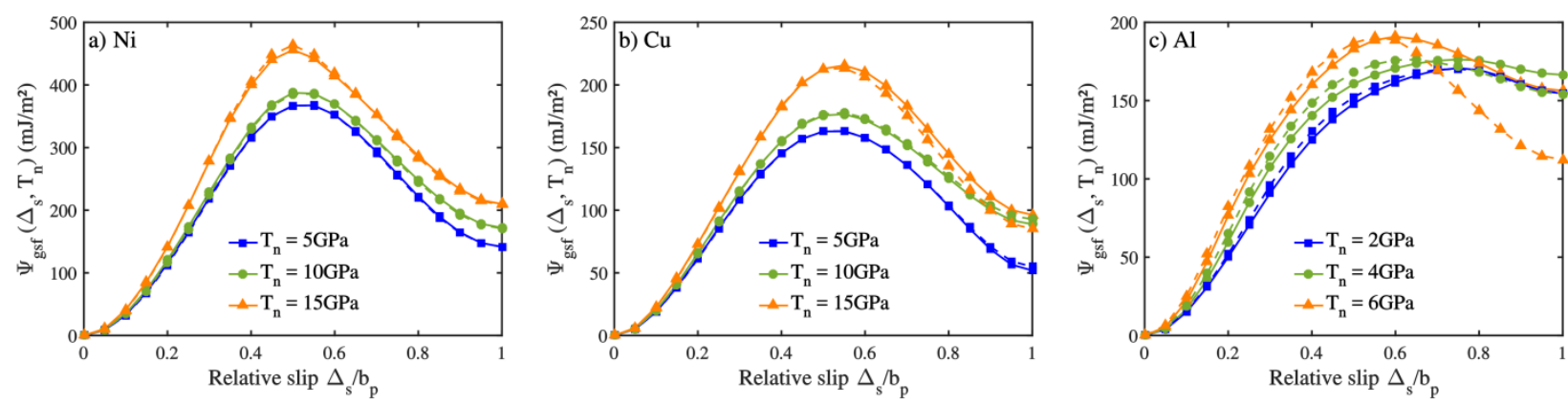

Figure 13: GSFE versus slip displacement, for various applied normal tensile stresses under uniaxial strain (solid line) and uniaxial stress (dashed line) as computed using interatomic potentials at $\mathrm{T}=0 \mathrm{~K}$ in a) nickel, b) copper and c) aluminum

\section{Appendix B. Effective elastic constants}

Our results in Section 5.2 are based on linear isotropic elasticity and thus require proper effective elastic constants input as a function of the normal stress applied. We use molecular statics simulations for calculating the effective elastic constants as follows. We define a simulation box, having periodic boundaries, oriented with $X=[\overline{1} 2 \overline{1}], Y=[111]$ and $Z=[10 \overline{1}]$, and with dimension of approximately $35 \times 60 \times 50 \AA$. We set a desired stress perpendicular to the $X-Z$ plane by increasing the lattice parameter along [111], while the lateral lattice parameter is held constant. At given applied normal stress we compute the effective shear modulus $\mu$ from the stress-shear strain response due to applied shear of $\tau_{y z}=0.1 M P a$. Then, by relaxing the stress along $\mathrm{Z}$ direction, we compute the effective Poisson's ratio as $v=-\varepsilon_{z z} / \varepsilon_{y y}$. Figure 14 shows the computed elastic constants as a function of applied stress in Ni and Al. For the applied stresses 
This is a post-print of the following article: Andric, Predrag; Yin, Binglun; Curtin, W.A. Journal of the Mechanics and Physics of Solids 2019,, 262-279.. The formal publication is available at http://dx.doi.org/10.1016/j.jmps.2018.09.007 @ 2019. This manuscript version is made available under the CC-BY-NC-ND 4.0 license http://creativecommons.org/licenses/by-nc-nd/4.0/

considered here we find that the shear modulus, in both studied materials, increases with normal tensile stress in part because the Poisson contraction decreases the in-plane atomic spacing which increases the curvature of the energy landscape in shear.
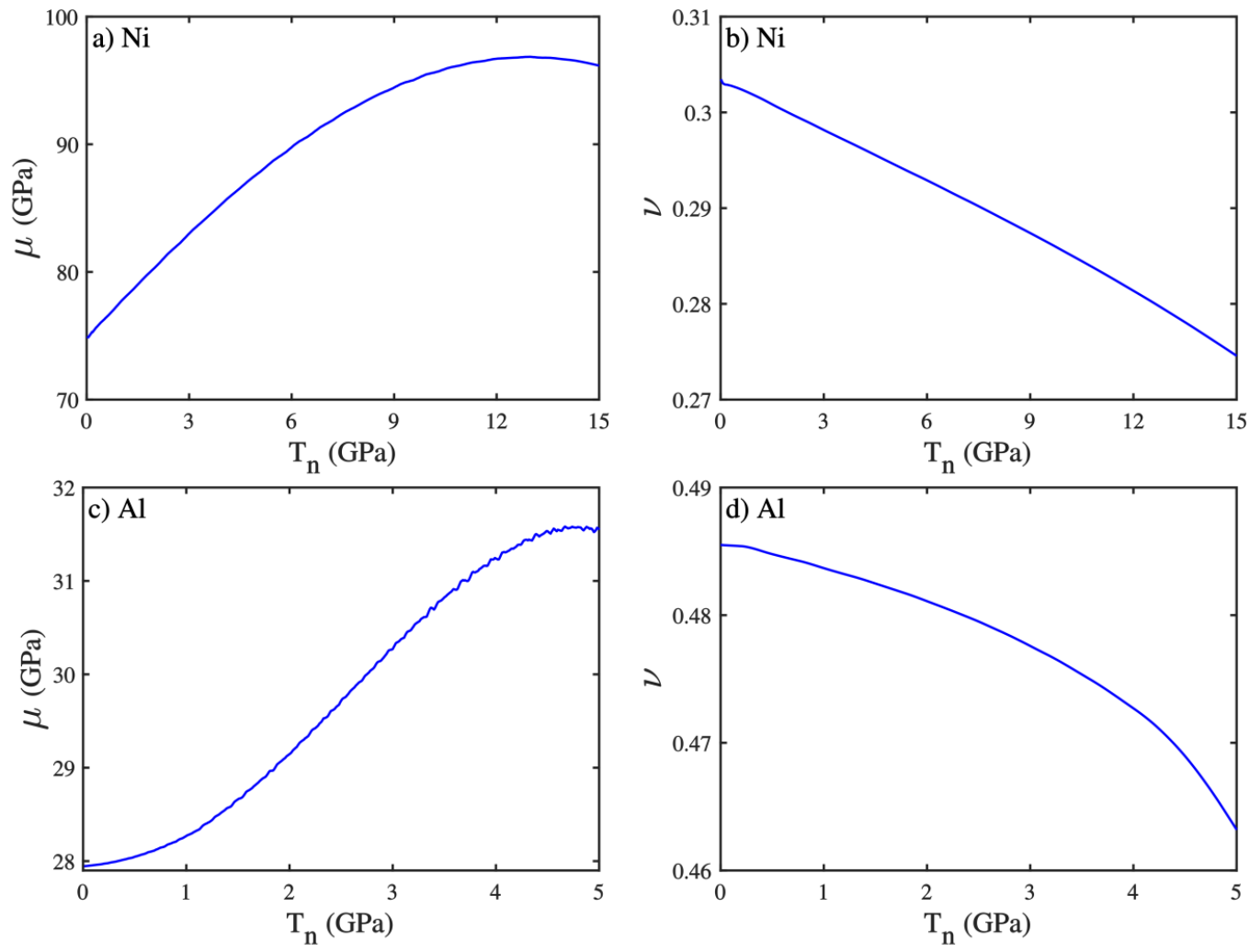

Figure 14: Effective elastic Shear modului and Poisson's ratios in Ni and Al computed using molecular statics at $\mathrm{T}=0 \mathrm{~K}$ as a function of the applied far-field normal stress.

Stress dependence of the materials elastic properties nominally affects only the interaction energy $W_{12}$ between two partials, since the inclusion energy $W_{I}$ is negligible. Therefore, we investigate how big this effect on predicted dissociation distance $d$ is. Figure 15 shows the simulated dissociation distance $d$, along with the predictions of Eq. 23 with and without taking into account stress dependence of the elastic properties. Influence of the stress dependent elastic properties on the overall behavior is more emphasized in $\mathrm{Ni}$ than in Al. The result sensitivity in Ni is due to similar contribution to the crystal enthalpy from $W_{12}$ and the stacking fault (change in GSFE and inelastic normal displacement with applied normal stress) itself. The stacking fault contribution in $\mathrm{Al}$ is bigger than $W_{12}$ (due to very big inelastic displacement as (see Figure 9)); thus, change in elastic properties only slightly influences predictions on the dissociation distance with increasing applied normal stress. 
This is a post-print of the following article: Andric, Predrag; Yin, Binglun; Curtin, W.A. Journal of the Mechanics and Physics of Solids 2019,, 262-279.. The formal publication is available at http://dx.doi.org/10.1016/j.jmps.2018.09.007 @ 2019. This manuscript version is made available under the CC-BY-NC-ND 4.0 license http://creativecommons.org/licenses/by-nc-nd/4.0/
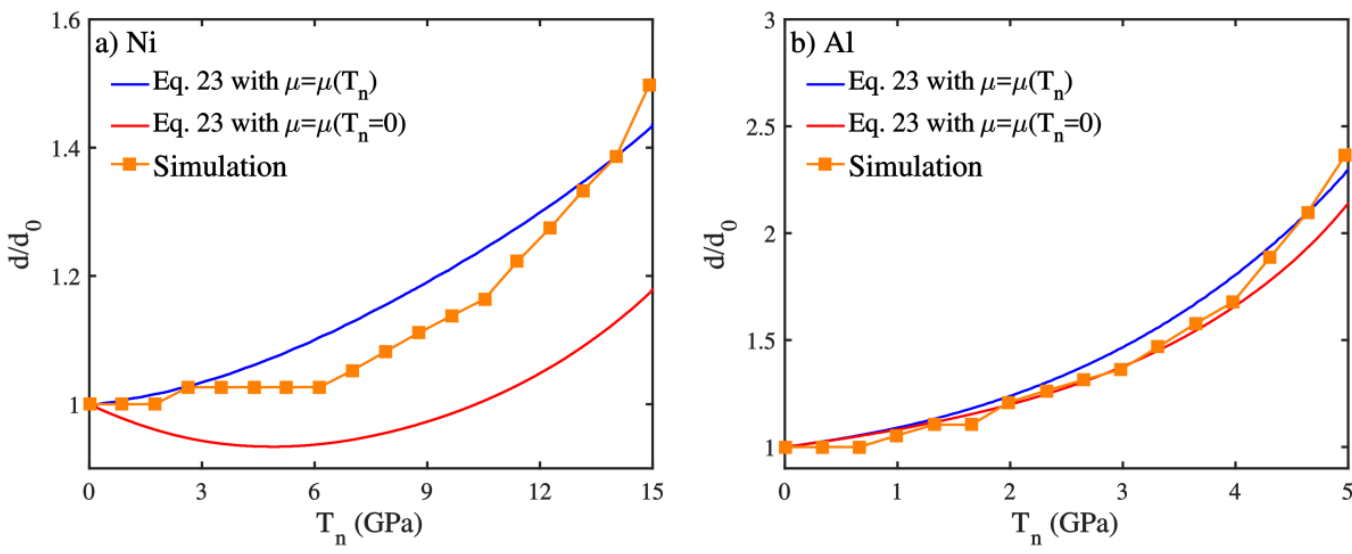

Figure 15: Equilibrium separation between partial dislocations in a) $\mathrm{Ni}$ and b) $\mathrm{Al}$ at different applied stress that perpendicular to the slip plane, normalized with its value at zero applied load, as predicted by equation by Eq. 23 when stress dependence of elastic properties is (blue color)/is not (red color) taken into account, and as observed in atomistic simulations (orange squares).

\section{References}

[1] Vitek V. Intrinsic stacking faults in body-centred cubic crystals. Philosophical Magazine. 1968 Oct

[2] Hirth, J.P., Lothe, J., 1982. Theory of Dislocations. Wiley, New York.

[3] Rice JR. Dislocation nucleation from a crack tip: an analysis based on the Peierls concept. Journal of the Mechanics and Physics of Solids. 1992 Jan 1;40(2):239-71.

[4] Andric P, Curtin WA. New Theory for Mode I Crack-tip Dislocation Emission. Journal of the Mechanics and Physics of Solids. 2017 Jun 15.

[5] Van Swygenhoven H, Derlet PM, Frøseth AG. Stacking fault energies and slip in nanocrystalline metals. Nature materials. 2004 Jun 1;3(6):399-403.

[6] Van Swygenhoven H. Grain boundaries and dislocations. Science. 2002 Apr 5;296(5565):66-7.

[7] Tadmor EB, Miller R, Phillips R, Ortiz M. Nanoindentation and incipient plasticity. Journal of Materials Research. 1999 Jun;14(6):2233-50.

[8] Tadmor EB, Hai S. A Peierls criterion for the onset of deformation twinning at a crack tip. Journal of the Mechanics and Physics of Solids. 2003 May 31;51(5):765-93.

[9] Andric P, Curtin WA. New theory for crack-tip twinning in fcc metals. Journal of Mechanics Physics of Solids. 2018 Apr;113:144-61.

665 [10] Sun Y, Beltz GE, Rice JR. Estimates from atomic models of tension-shear coupling in dislocation nucleation from a crack tip. Materials Science and Engineering: A. 1993 Oct 1;170(1-2):67-85.

[11] Mishin Y, Farkas D, Mehl MJ, Papaconstantopoulos DA. Interatomic potentials for monoatomic metals from experimental data and ab initio calculations. Physical Review B. 1999 Feb 1;59(5):3393.

[12] Zhou SJ, Carlsson AE, Thomson R. Crack blunting effects on dislocation emission from cracks. Physical review letters. $1994 \mathrm{Feb} 7 ; 72(6): 852$.

[13] Knap J, Sieradzki K. Crack tip dislocation nucleation in FCC solids. Physical review letters. 1999 Feb 22;82(8):1700.

[14] Zimmerman JA, Gao H, Abraham FF. Generalized stacking fault energies for embedded atom FCC metals. Modelling and Simulation in Materials Science and Engineering. 2000 Mar;8(2):103.

[15] Möller JJ, Bitzek E. Comparative study of embedded atom potentials for atomistic simulations of fracture in $\alpha$ iron. Modelling and Simulation in Materials Science and Engineering. 2014 Apr 11;22(4):045002.

[16] Brandl C, Derlet PM, Van Swygenhoven H. General-stacking-fault energies in highly strained metallic environments: Ab initio calculations. Physical Review B. 2007 Aug 29;76(5):054124.

[17] Branicio PS, Zhang JY, Srolovitz DJ. Effect of strain on the stacking fault energy of copper: a first-principles study. Physical Review B. 2013 Aug 26;88(6):064104.

[18] Plimpton S. Fast parallel algorithms for short-range molecular dynamics. Journal of computational physics. 1995 Mar 1;117(1):1-9.

[19] Kresse G, Furthmüller J. Efficient iterative schemes for ab initio total-energy calculations using a plane-wave basis set. Physical review B. 1996 Oct 15;54(16):11169.

[20] Kresse G, Joubert D. From ultrasoft pseudopotentials to the projector augmented-wave method. Physical Review B. 1999 Jan 15;59(3):1758. 
This is a post-print of the following article: Andric, Predrag; Yin, Binglun; Curtin, W.A. Journal of the Mechanics and Physics of Solids 2019,, 262-279.. The formal publication is available at http://dx.doi.org/10.1016/j.jmps.2018.09.007 @ 2019. This manuscript version is made available under the CC-BY-NC-ND 4.0 license http://creativecommons.org/licenses/by-nc-nd/4.0/

[21] Bitzek E, Koskinen P, Gähler F, Moseler M, Gumbsch P. Structural relaxation made simple. Physical review letters. 2006 Oct 27;97(17):170201

[22] Mishin Y, Mehl MJ, Papaconstantopoulos DA, Voter AF, Kress JD. Structural stability and lattice defects in copper: Ab initio, tight-binding, and embedded-atom calculations. Physical Review B. 2001 May 21;63(22):224106.

[23] Perdew JP, Burke K, Ernzerhof M. Generalized gradient approximation made simple. Physical review letters. 1996 Oct 28;77(18):3865.

[24] Blöchl PE. Projector augmented-wave method. Physical review B. 1994 Dec 15;50(24):17953.

695 [25] Kibey S, Liu JB, Johnson DD, Sehitoglu H. Generalized planar fault energies and twinning in Cu-Al alloys. Applied Physics Letters. 2006 Nov 6;89(19):191911.

[26] Yin B, Wu Z, Curtin WA. Comprehensive first-principles study of stable stacking faults in hcp metals. Acta Materialia. 2017 Jan 15;123:223-34.

[27] Eshelby JD. The determination of the elastic field of an ellipsoidal inclusion, and related problems. InProceedings of the Royal Society of London A: Mathematical, Physical and Engineering Sciences 1957 Aug 20 (Vol. 241, No. 1226, pp. 376-396). The Royal Society.

[28] Mura T. Micromechanics of defects in solids. Springer Science \& Business Media; 2013 Mar 9.

[29] Osetsky YN, Bacon DJ. An atomic-level model for studying the dynamics of edge dislocations in metals. Modelling and simulation in materials science and engineering. 2003 May 1;11(4):427.

705 [30] Stukowski A. Visualization and analysis of atomistic simulation data with OVITO-the Open Visualization Tool. Modelling and Simulation in Materials Science and Engineering. 2009 Dec 15;18(1):015012. 